\title{
Rényi entropies of free bosons on the torus and holography
}

\section{Shouvik Datta and Justin R. David}

Centre for High Energy Physics, Indian Institute of Science, C.V. Raman Avenue, Bangalore 560012, India

E-mail: shouvik@cts.iisc.ernet.in, justin@cts.iisc.ernet.in

ABSTRACT: We analytically evaluate the Rényi entropies for the two dimensional free boson CFT. The CFT is considered to be compactified on a circle and at finite temperature. The Rényi entropies $S_{n}$ are evaluated for a single interval using the two point function of bosonic twist fields on a torus. For the case of the compact boson, the sum over the classical saddle points results in the Riemann-Siegel theta function associated with the $A_{n-1}$ lattice. We then study the Rényi entropies in the decompactification regime. We show that in the limit when the size of the interval becomes the size of the spatial circle, the entanglement entropy reduces to the thermal entropy of free bosons on a circle. We then set up a systematic high temperature expansion of the Rényi entropies and evaluate the finite size corrections for free bosons. Finally we compare these finite size corrections both for the free boson CFT and the free fermion CFT with the one-loop corrections obtained from bulk three dimensional handlebody spacetimes which have higher genus Riemann surfaces as its boundary. One-loop corrections in these geometries are entirely determined by quantum numbers of the excitations present in the bulk. This implies that the leading finite size corrections contributions from one-loop determinants of the Chern-Simons gauge field and the Dirac field in the dual geometry should reproduce that of the free boson and the free fermion CFT respectively. By evaluating these corrections both in the bulk and in the CFT explicitly we show that this expectation is indeed true.

Keywords: AdS-CFT Correspondence, Conformal Field Models in String Theory

ARXIV EPRINT: 1311.1218 


\section{Contents}

1 Introduction 1

2 Bosonic twist correlators on the torus 5

2.1 Quantum contribution 8

$\begin{array}{ll}2.2 \text { Classical contribution } & 12\end{array}$

2.3 Integrals of the cut differentials 14

$\begin{array}{ll}2.4 \text { Rényi entropies of a compact boson } & 16\end{array}$

3 Rényi entropies for a non-compact boson $\quad 19$

$\begin{array}{lll}3.1 & \text { Plots } & 20\end{array}$

3.2 Thermal entropy from entanglement entropy 21

4 Finite size corrections to Rényi entropies $\quad 24$

$\begin{array}{lll}4.1 \text { Bosons } & 26\end{array}$

$\begin{array}{lll}4.2 & \text { Fermions } & 27\end{array}$

5 Finite size corrections from holography $\quad 29$

5.1 One-loop corrections: bosons 33

5.2 One loop corrections: fermions 34

5.3 Discussion 36

$\begin{array}{lll}6 & \text { Conclusions } & 36\end{array}$

$\begin{array}{ll}\text { A Jacobi theta functions } & 38\end{array}$

$\begin{array}{lr}\text { B Evaluating } W_{1}^{1} \text { and } W_{2}^{2} & 39\end{array}$

C One-loop determinant for the Chern-Simons gauge field 46

$\begin{array}{ll}\text { D Sums involving 2-letter words } & 47\end{array}$

\section{Introduction}

Entanglement entropy is emerging as an important observable to characterize behaviour of quantum field theories and many body theories. In condensed matter physics entanglement entropy is used as an order parameter to characterize quantum phase transitions [1]. ${ }^{1}$ It is also useful as a measure of thermalization in non-equilibrium statistical mechanics [3]. Entanglement entropy is defined as follows [4-6]: first partition the complete set of observables

\footnotetext{
${ }^{1}$ See [2] for a complete list of references for the application of entanglement entropy to many body physics.
} 
of the quantum systems into two disjoint Hilbert spaces $A$ and its complement $B$. Define the reduced density matrix $\rho_{A}$ by tracing over the observables in $B$. Then entanglement entropy between the observables in the two Hilbert spaces is defined by

$$
S_{E}=\operatorname{Tr}\left(\rho_{A} \ln \rho_{A}\right) .
$$

In quantum field theories usually the Hilbert spaces $A$ refers to the degrees of freedom in a spatial region $A$. Evaluation of entanglement entropy is usually done by computing the Rényi entropies which are defined as

$$
S_{n}=-\frac{1}{n-1} \ln \operatorname{Tr}\left(\rho_{A}^{n}\right) .
$$

Then entanglement entropy is then derived as the limit $S_{E}=\lim _{n \rightarrow 1} S_{n}$.

Though entanglement entropy has proved to be an useful observable, it is difficult to compute even in the case of free theories. In $1+1$ dimensional conformal field theories if $A$ denotes a single interval of length $L$, the evaluation of Rényi entropies can be done exactly using the replica trick [6-9]. The Rényi entropies $S_{n}$ are determined by evaluating the partition function of the CFT on a $n$ Riemann sheets joined consecutively and cyclically along the interval $A$. In general these Riemann sheets form a higher genus surface. This can then be shown to be equivalent to evaluating the two point function of the 'twist' operator of dimension

$$
\Delta_{n}=\bar{\Delta}_{n}=\frac{c}{24}\left(n-\frac{1}{n}\right),
$$

inserted at the end points of the interval. Here $c$ refers to the central charge of the CFT. We therefore obtain

$$
\begin{aligned}
\exp \left(-(n-1) S_{n}(A)\right) & =\left\langle\mathcal{T}_{n}(L) \overline{\mathcal{T}}_{n}(0)\right\rangle \\
& =\left(\frac{a}{L}\right)^{4 \Delta_{n}}
\end{aligned}
$$

where $\mathcal{T}_{n}$ is the twist operator and $a$ the cut-off. We have normalized the two point functions to unity. Change in this normalization just leads to a non-universal constant. Thus the Rényi entropies for a single interval are given by

$$
S_{n}(A)=\frac{c(n+1)}{6 n} \ln \frac{L}{a} .
$$

Though these are evaluated for an integer $n$, the resulting expression is assumed to be analytic in $n$ and the entanglement entropy is obtained in the $n \rightarrow 1$ limit. Note that the only information Rényi entropies of a single interval carry about the conformal field theory is its central charge.

There are two possible methods to obtain more information regarding the details of the CFT using Rényi entropies. One is to generalize the subsystem $A$ to multiple intervals [10-13]. The other is to consider the CFT on a circle of radius $R$ and at finite temperature $T$, that is the CFT is on a torus. Rényi entropies for CFT's on tori contain finite size corrections which are suppressed by $\mathcal{O}\left(e^{-\pi R T}\right)$ in the high temperature expansion compared to the result in (1.5). These depend on the details of the CFT. In this 
regard, the only known analytical result for Rényi entropies is the case of free fermions on the torus $[14,15]$. In this paper we generalize this to the case of a free complex boson on the torus. ${ }^{2}$ Rényi entropies corresponding to a single interval is evaluated by using the two point function of the bosonic twist operators on the torus. These two point functions can be evaluated by the 'stress-tensor method' [17-19] (see also [20]). As far as we are aware this is the first instance of an application of the bosonic twist correlators on the torus. The Rényi entropies, $S_{n}$ consists of two contributions one due to the quantum part of the two point function and the other due to the various saddle points of the classical action. For a boson compactified on a spacetime square torus of size $\mathcal{R}$ and for a rectangular worldsheet torus we show that the contribution of the classical saddle points to the two point function reduces to the Riemann-Seigel theta function associated with the $A_{n-1}$ lattice. For the interested reader the result is given in (2.89). The compact free boson CFT describes a class of $1+1$ dimensional condensed matter systems called Luttinger liquids. Examples of these are Heisenberg spin chains and 1-dimensional Bose gases with repulsive interactions. At the conformal point they can be described by compact bosons but with different radius $\mathcal{R}$. Therefore our result is relevant for all these systems.

We then study the de-compactification regime in detail. The result for the Rényi entropies in this regime is given in (3.2). We first show that when the size of the interval $L$ approaches the size of the circle $R$, the entanglement entropy $S_{1}$ reduces to the thermal entropy of a complex boson on a circle. This serves a simple check of our result. We then set up a systematic high temperature expansion of the Rényi entropies and obtain the leading finite size corrections.

The second goal of this paper is to compare the finite size corrections for both the free boson theory and for the free fermion theory to that obtained from holography. Though entanglement entropy is a difficult quantity to evaluate in field theories, there is a very simple proposal by Ryu and Takanayagi to evaluate it in strongly coupled field theories which admit a gravitational dual [21,22]. Recently for the case of conformal field theories in 2 dimensions which admit a gravitational dual, the Ryu-Takanayagi proposal has been derived from the conventional rules of $A d S / C F T$ [23]. This derivation has been extended to higher dimensions in [24]. Recall that Rényi entropies are determined by evaluating the partition function of the CFT on a $n$-sheeted Riemann surface. The $n$-sheets are joined consecutively and cyclically along the interval $A$. From the rules of $A d S / C F T$ we obtain

$$
\begin{aligned}
Z_{n}(C F T) & =\exp \left(-(n-1) S_{n}(A)(C F T)\right)=\int \mathcal{D}[g] e^{-\mathcal{S}[g]}, \\
& =\exp \left(c \mathcal{S}_{\text {classical }}+\mathcal{S}_{\text {1-loop }}+\mathcal{O}\left(\frac{1}{c}\right)\right)
\end{aligned}
$$

where $g$ refers to all fields which occur in the bulk action. The path integral over $g$ is done over bulk geometries whose boundaries reduce to the $n$-sheeted Riemann surface which on which the CFT lives. $\mathcal{S}_{\text {classical }}$ is obtained by evaluating the classical action on these bulk geometries. $c$ is the central charge which is related to the inverse of the three dimensional

\footnotetext{
${ }^{2}$ Numerical results for the entanglement entropy of the massive scalar in $1+1$ dimensions were obtained in $[16]$.
} 
Newton's constant. $\mathcal{S}_{1 \text {-loop }}$ refers to the one-loop contribution on performing the path integral. Explicit bulk duals of CFT's living on the replica geometry which in general is a higher genus Riemann surface were constructed in [23]. These bulk duals are handlebody geometries which can be constructed by realizing the higher genus Riemann surface at the boundary using Schottky uniformization [25]. The partition function of the CFT is evaluated holographically by computing the path integral in (1.6). Using the equality in (1.6) the leading contribution of the Rényi entropies at large $c$ can be obtained by evaluating $\mathcal{S}_{\text {classical }}$. In the $n \rightarrow 1$ limit it has been shown to reduce to the Ryu-Takanayagi prescription.

Using this approach a method to obtain one-loop corrections to the Rényi entropies as well as the Ryu-Takanayagi formula has been proposed in [26]. ${ }^{3}$ The proposal has been verified upto the 6th order in the short interval expansion of the Renyi entropy of two disjoint intervals on a plane in [29]. This proposal also predicts finite size corrections to the Rényi entropies of a single interval on a torus. Determining the 1-loop corrections $\mathcal{S}_{1 \text {-loop }}$ involves evaluating one-loop determinants of all the fields present in the bulk handlebody geometries. One loop determinants in these handlebody geometries have been evaluated in [31] following a conjecture by [30]. The one-loop determinants are sensitive to only the quantum numbers of the spectrum of the fields in the bulk. That is, given the spin and the mass of the bulk field, the one-loop determinant is completely specified. One-loop determinants are in-sensitive to interactions. In fact the determinant organizes itself into characters of the Virasoro algebra as expected from symmetry considerations Further more the 1-loop contribution is independent of the central charge of the theory. In fact when the bulk geometry is thermal $A d S_{3}$ which is dual to the CFT on a genus one surface, it has been seen that the 1-loop determinant of a single field in the bulk organizes it self into Virasoro blocks and is equal to the partition function of the dual field in the CFT [31, 35-37]. Note that the dual operator is also entirely determined by the quantum numbers of the corresponding field in the bulk. Thus we expect the contribution to the finite size corrections of the Rényi entropies from the 1-loop determinant of a single U(1) l Chern-Simons should capture the leading finite size corrections of the Rényi entropies of single boson. The Chern-Simons field in the bulk is dual to currents with conformal dimensions $(1,0)$ and $(0,1)$ which are the physical degrees of freedom of a free boson CFT. Similarly we expect the leading contribution to the finite size corrections of the Rényi entropies from the 1-loop determinant of a single Dirac field to be captured by the free fermions with conformal dimensions $(1 / 2,0)$ and $(0,1 / 2)$. It is important to note that the classical action $\mathcal{S}_{\text {classical }}$ also contains finite size corrections to Rényi entropies. We will see that these finite size corrections are sub-leading in $e^{-\pi R T}$ when compared to that from the one loop determinants for both the Chern-Simons field as well as the Dirac field in the bulk. These finite size corrections also do not contribute to the entanglement entropy as they vanish in the $n \rightarrow 1$ limit. $^{4}$

\footnotetext{
${ }^{3}$ An alternate method to evaluate one loop corrections has been proposed by [27] for higher dimensions. This has been verified recently for the case of 3 dimensional CFT's in [28].

${ }^{4}$ The finite size contributions to the Rényi entropies from the 1-loop determinant of the graviton in the bulk begins at the same order in $e^{-\pi R T}$ as the finite size contributions from $\mathcal{S}_{\text {classical }}$. Therefore in this case the leading finite size corrections cannot be obtained just from $\mathcal{S}_{1 \text {-loop }}$.
} 
Motivated by these arguments we evaluate the leading finite size corrections obtained by considering the one loop determinant of the Chern-Simons gauge field in the handlebody geometries. We show that these corrections agree precisely with the leading finite size corrections for the case of free bosons on the torus. We also compare the finite size corrections obtained by considering the one loop determinant of the Dirac field on the handle body geometries and obtain precise agreement with the leading finite size corrections for the case of free fermions on the torus. We consider these agreements as a non-trivial check of the method proposed in [26].

The organization of the paper is as follows. In the next section we derive the Rényi entropies of a free compact boson for a single interval on a torus. We detail the necessary methods from the theory of $\mathbb{Z}_{n}$ orbifolds for this purpose. In section 3 we discuss the decompactification regime in detail and show that in the limit when the size of the interval becomes the size of the CFT spatial circle the entanglement entropy reduces to the thermal entropy of bosons on a circle. In section 4 we write down the leading finite size corrections to Rényi entropies for free bosons on the torus and for the free fermions in the high temperature expansion. The result for the free fermions has been derived earlier in [14] and we review it here for completeness. In section 5 we evaluate finite size corrections to Rényi entropies in the bulk handlebody geometries using the method proposed in [26]. We show that finite size corrections obtained by considering one-loop determinants of the Chern-Simons gauge field and the Dirac field agree precisely with the leading finite corrections obtained for the free boson and free fermion CFT. Finally, section 6 contains our conclusions. Appendix A contains details of conventions we use for Jacobi-theta functions. Appendix B derives various identities for integrals of cut differentials used in the main text. Appendix C contains the evaluation of the one-loop determinant of the Chern-Simons gauge field in $A d S_{3}$. Appendix D derives certain sums which are used for evaluating the one-loop determinants in handlebody geometries.

\section{Bosonic twist correlators on the torus}

The replica trick reduces the evaluation of the Renyi entropy for a single interval in $1+1$ CFT's to the computation of the two point function of twist fields. The CFT of interest is the free complex boson CFT in $1+1$ dimensions, the complex boson is compactified on square torus of radius $\mathcal{R}$. It obeys the boundary conditions

$$
\phi\left(e^{2 \pi i} z, e^{-2 \pi i} \bar{z}\right)=\phi(z, \bar{z})+\mathcal{R}\left(m_{1}+i m_{2}\right),
$$

The system is of finite size and is kept at finite temperature $\beta$. Let $\phi_{i}$ with $i=0,1, \cdots, n-1$ label the replica copies. To diagonalize the action of the cyclic permutation of the copies we consider the following linear combinations

$$
\tilde{\phi}_{k}=\sum_{j=0}^{n-1} e^{2 \pi i j \frac{k}{n}} \phi_{j}, \quad k=0, \cdots n-1 .
$$

$\tilde{\phi}_{k}$ is multiplied by the phase $e^{2 \pi i \frac{k}{n}}$ as one moves around the twist operator. The boundary conditions in (2.1) also need to be imposed on $\tilde{\phi}_{k}$. Thus we obtain the following boundary 
conditions on $\tilde{\phi}_{k}$

$$
\tilde{\phi}_{k}\left(e^{2 \pi i} z, e^{-2 \pi i} \bar{z}\right)=e^{2 \pi i \frac{k}{n}} \tilde{\phi}_{k}(z, \bar{z})+\mathcal{R} \sum_{j=0}^{n-1} e^{2 \pi i j \frac{k}{n}}\left(m_{j, 1}+i m_{j, 2}\right) .
$$

Thus $\tilde{\phi}_{k}$ is compactified on the lattice $\Lambda_{\frac{k}{n}}$ which is defined as

$$
\Lambda_{\frac{k}{n}} \equiv\left\{q=\mathcal{R} \sum_{j=0}^{n-1} e^{\frac{2 \pi i k j}{n}}\left(m_{j, 1}+i m_{j, 2}\right) ; m_{j, 1}, m_{j, 2} \in \mathbf{Z}\right\} .
$$

We now split $\tilde{\phi}_{k}$ into the classical and the quantum part as $\tilde{\phi}_{k}=\tilde{\phi}_{k}^{q u}+\tilde{\phi}_{k}^{c l}$. The two point function of the twist fields then splits into the a contribution from the quantum part and the sum over classical saddle points. The classical part obeys the boundary condition

$$
\tilde{\phi}_{k}^{c l}\left(e^{2 \pi i} z, e^{-2 \pi i} \bar{z}\right)=e^{2 \pi i \frac{k}{n}} \tilde{\phi}_{k}^{c l}(z, \bar{z})+v,
$$

where $v$ is a vector in the lattice $\Lambda_{\frac{k}{n}}$. The quantum part obeys the boundary condition

$$
\tilde{\phi}_{k}^{q u}\left(e^{2 \pi i} z, e^{-2 \pi i} \bar{z}\right)=e^{2 \pi i \frac{k}{n}} \tilde{\phi}_{k}^{q u}(z, \bar{z}) .
$$

Let $\sigma_{k, n}$ be the twist field which responsible for the boundary condition in (2.3) and $\bar{\sigma}_{k, n}$ the anti-twist field. Let the two point function of these operators on the torus be given by

$$
Z_{k, n}=\left\langle\sigma_{k, n}\left(z_{1}, \bar{z}_{1}\right) \bar{\sigma}_{k, n}\left(z_{2}, \bar{z}_{2}\right)\right\rangle
$$

Then the Rényi entropy on the torus is given by

$$
\exp \left((1-n) S_{n}\right)=\prod_{k=0}^{n-1} Z_{k, n}=\prod_{k=0}^{n-1}\left\langle\sigma_{k, n}\left(z_{1}, \bar{z}_{1}\right) \bar{\sigma}_{k, n}\left(z_{2}, \bar{z}_{2}\right)\right\rangle .
$$

From this discussion, we see that the first task is to evaluate the two point function of the twist operators on the torus. This has been done in [18] and for the $n=2$ case by [19]. To make our discussion self contained and simplified we review the procedure to evaluate this two point function. To un-clutter the notation, let the complex boson be denoted by $X$. Then the steps to obtain the two point function are as follows.

1. We first write down the Greens functions

$$
\begin{aligned}
& g\left(z, w ; z_{i}, \bar{z}_{i}\right) \equiv \frac{1}{Z_{k, n}}\left\langle-\partial_{z} X \partial_{w} \bar{X} \sigma_{k, n}\left(z_{1}, \bar{z}_{1}\right) \bar{\sigma}_{k, n}\left(z_{2}, \bar{z}_{2}\right)\right\rangle \\
& h\left(\bar{z}, w ; z_{i}, \bar{z}_{i}\right) \equiv \frac{1}{Z_{k, n}}\left\langle-\partial_{\bar{z}} X \partial_{w} \bar{X} \sigma_{k, n}\left(z_{1}, \bar{z}_{1}\right) \bar{\sigma}_{k, n}\left(z_{2}, \bar{z}_{2}\right)\right\rangle .
\end{aligned}
$$

where $z_{i}, i=1,2$ are the two points at which the twist operators are inserted. These correlators are determined using the behaviour as $z, w$ approach the points at which the twist fields are inserted and monodromy conditions. 
2. The monodromy conditions are given as follows. First split the $X$ into a classical piece and a quantum fluctuation

$$
X=X_{c l}+X_{q u}
$$

When integrated over closed loops over the two cycles of the torus the quantum piece does not change, that is

$$
\Delta_{\gamma_{a}} X_{q u}=\oint_{\gamma_{a}} d z \partial_{z} X_{q u}+\oint_{\gamma_{a}} d \bar{z} \partial_{\bar{z}} X_{q u}=0
$$

Here $\gamma_{a}, a=1,2$ are the two cycles of the torus. The classical part obeys

$$
\Delta_{\gamma_{a}} X_{c l}=\oint_{\gamma_{a}} d z \partial_{z} X_{c l}+\oint_{\gamma_{a}} d \bar{z} \partial_{\bar{z}} X_{c l}=v_{a}
$$

where $v_{a}$ is related to a translation on the compact torus $\Lambda_{\frac{k}{n}}$, which will be specified subsequently.

3. The Green's functions $g\left(z, w ; z_{i}, \bar{z}_{i}\right), h\left(\bar{z}, w ; z_{i}, \bar{z}_{i}\right)$ are written down which obey the monodromy conditions and have the right singularity structure when $z, w$ approaches the twist insertions $z_{i}, \bar{z}_{i}$. Then we obtain the expectation value of the stress tensor by taking the following limit

$$
\langle T(z)\rangle=\lim _{\omega \rightarrow z}\left(g(z, w)-\frac{1}{(z-w)^{2}}\right) .
$$

4. The OPE of the stress tensor with the primary field $\phi$ of weight $h_{\phi}$ is given by

$$
T(z) \phi(w, \bar{w}) \sim \frac{h_{\phi}}{(z-w)^{2}} \phi(w, \bar{w})+\frac{1}{(z-w)} \partial_{w} \phi(w, \bar{w}) .
$$

Using this OPE we can obtain the following set of differential equations for the two point function contribution from the quantum part $Z_{k, n}^{q u}$.

$$
\partial_{z_{i}} \ln Z_{k, n}^{q u}=\lim _{z \rightarrow z_{i}}\left(\left(z-z_{i}\right)\langle T(z)\rangle-\frac{h}{\left(z-z_{i}\right)}\right)
$$

where

$$
h=\frac{1}{2} \frac{k}{n}\left(1-\frac{k}{n}\right),
$$

is the weight of the twist field. This can be repeated and a similar set of differential equations can be obtained by considering the OPE of the anti-holomorphic stress tensor $\bar{T}(\bar{z})$ with the twist fields.

5. Solving these differential equations result in the quantum part of the two point function $Z_{k, n}^{q u}$. 
6. The classical part of the two point function is obtained by considering the classical solutions $\partial_{z} X_{c l}, \partial_{\bar{z}} X_{c l}$ which satisfy the monodromy conditions given in (2.11). We then evaluate the classical action given by

$$
S_{c l}\left(X_{c l}, X_{c l}\right)=\frac{1}{4 \pi} \int d^{2} z\left(\partial_{z} X \partial_{\bar{z}} \bar{X}+\partial_{\bar{z}} X \partial_{z} \bar{X}\right) .
$$

7. The full two point function is then given by summing over all the classical solutions

$$
Z=Z_{k, n}^{q u} \sum_{\left\langle X_{c l}\right\rangle} e^{-S_{c l}}
$$

\subsection{Quantum contribution}

In this subsection we construct the quantum contribution $Z_{k, n}^{q u}$ of the two point function of the twist operators. We begin with writing down the the Green's function $g$ and $h$ which satisfy the required conditions. To do this we write down the cut differential for $\left\langle\partial_{z} X\right\rangle$, where the expectation value is taken in presence of the twist operators. The twist operator is located at $z_{1}$ while the anti-twist operator is located at $z_{2}$. This is given by

$$
\omega_{1}(z)=\vartheta_{1}\left(z-z_{1}\right)^{-(1-k / n)} \vartheta_{1}\left(z-z_{2}\right)^{-k / n} \vartheta_{1}\left(z-(1-k / n) z_{1}-(k / n) z_{2}\right) .
$$

Here $z_{1}$ is the location of the twist operator and $z_{2}$ is the location of the anti-twist operator. Note that these cut differentials are doubly periodic, that is they are periodic under $z \rightarrow$ $z+1$ and $z \rightarrow z+\tau$ where $\tau$ is the modular parameter of the torus. They also have the required behaviour

$$
\begin{aligned}
\lim _{z \rightarrow z_{1}}\left\langle\partial_{z} X\right\rangle & \sim\left(z-z_{1}\right)^{-\left(1-\frac{k}{n}\right)}+\cdots, \\
\lim _{z \rightarrow z_{2}}\left\langle\partial_{z} X\right\rangle & \sim\left(z-z_{2}\right)^{-k / n}+\cdots .
\end{aligned}
$$

We also have the cut differential for $\left\langle\partial_{z} \bar{X}\right\rangle$ which is given by

$$
\omega_{2}(z)=\vartheta_{1}\left(z-z_{1}\right)^{-k / n} \vartheta_{1}\left(z-z_{2}\right)^{-(1-k / n)} \vartheta_{1}\left(z-\frac{k}{n} z_{1}-\left(1-\frac{k}{n}\right) z_{2}\right) .
$$

Again this differential is doubly periodic, and it has the required behaviour

$$
\begin{aligned}
& \lim _{z \rightarrow z_{1}}\left\langle\partial_{\bar{z}} X\right\rangle \sim\left(z-z_{1}\right)^{-k / n}+\cdots, \\
& \lim _{z \rightarrow z_{2}}\left\langle\partial_{\bar{z}} X\right\rangle \sim\left(z-z_{2}\right)^{-(1-k / n)}+\cdots .
\end{aligned}
$$

The cut differentials for $\left\langle\partial_{\bar{z}} X\right\rangle$ is given by the complex conjugate

$$
\bar{\omega}_{2}(\bar{z})=\vartheta_{1}\left(\bar{z}-\bar{z}_{1}\right)^{-k / n} \vartheta_{1}\left(\bar{z}-\bar{z}_{2}\right)^{-(1-k / n)} \vartheta_{1}\left(\bar{z}-\frac{k}{n} \bar{z}_{1}-\left(1-\frac{k}{n}\right) \bar{z}_{2}\right) .
$$

This is again doubly periodic and has the behaviour

$$
\begin{aligned}
& \lim _{\bar{z} \rightarrow \bar{z}_{1}}\left\langle\partial_{\bar{z}} X\right\rangle \sim\left(\bar{z}-\bar{z}_{1}\right)^{-k / n}+\cdots, \\
& \lim _{\bar{z} \rightarrow \bar{z}_{1}}\left\langle\partial_{\bar{z}} X\right\rangle \sim\left(\bar{z}-\bar{z}_{2}\right)^{-(1-k / n)}+\cdots .
\end{aligned}
$$


Similarly the cut differential for $\left\langle\partial_{\bar{z}} \bar{X}\right\rangle$ is given by

$$
\bar{\omega}_{1}(\bar{z})=\vartheta_{1}\left(\bar{z}-\bar{z}_{1}\right)^{-(1-k / n)} \vartheta_{1}\left(\bar{z}-\bar{z}_{2}\right)^{-k / n} \vartheta_{1}\left(\bar{z}-\left(1-\frac{k}{n}\right) \bar{z}_{1}-\frac{k}{n} \bar{z}_{2}\right) .
$$

This has the behaviour

$$
\begin{aligned}
& \lim _{\bar{z} \rightarrow \bar{z}_{1}}\left\langle\partial_{\bar{z}} X\right\rangle \sim\left(\bar{z}-\bar{z}_{1}\right)^{-(1-k / n)}+\cdots, \\
& \lim _{\bar{z} \rightarrow \bar{z}_{1}}\left\langle\partial_{\bar{z}} X\right\rangle \sim\left(\bar{z}-\bar{z}_{2}\right)^{-k / n}+\cdots .
\end{aligned}
$$

Let us also define

$$
\begin{aligned}
\gamma_{1-k / n} & =\vartheta_{1}\left(z-z_{1}\right)^{-(1-k / n)} \vartheta_{1}\left(z-z_{2}\right)^{-k / n)}, \\
\gamma_{k / n} & =\vartheta_{1}\left(z-z_{1}\right)^{-k / n} \vartheta_{1}\left(z-z_{2}\right)^{-(1-k / n)}, \\
Y_{1} & =-\left(1-\frac{k}{n}\right)\left(z_{1}-z_{2}\right), \\
Y_{2} & =\frac{k}{n}\left(z_{1}-z_{2}\right),
\end{aligned}
$$

to un-clutter our expressions.

We can now write down the Green's functions.

$$
\begin{aligned}
& g(z, w)=g_{s}(z, w)+A \omega_{1}(z) \omega_{2}(w), \\
& h(z, w)=B \bar{\omega}_{2}(\bar{z}) \omega_{2}(w) .
\end{aligned}
$$

$A, B$ will be determined by the monodromy conditions given in (2.11). $g_{s}$ contains the double pole in the respective Green's function and is given by

$$
g_{s}(z, w)=\gamma_{1-k / n}(z) \gamma_{k / n}(w)\left(\frac{\vartheta_{1}^{\prime}(0)}{\vartheta_{1}(z-w)}\right)^{2} P(z, w)
$$

where

$$
\begin{aligned}
P(z, w) & =\frac{k}{N} F_{1}(z, w) \vartheta_{1}\left(w-z_{1}\right) \vartheta\left(z-z_{2}\right)+\left(1-\frac{k}{N}\right) F_{2}(z, w) \vartheta_{1}\left(w-z_{2}\right) \vartheta\left(z-z_{1}\right) \\
F_{1}(z, w) & =\frac{\vartheta_{1}\left(z-w+U_{1}\right)}{\vartheta_{1}\left(U_{1}\right)} \frac{\vartheta_{1}\left(z-w+Y_{1}-U_{1}\right)}{\vartheta_{1}\left(Y_{1}-U_{1}\right)} \\
F_{2}(z, w) & =\frac{\vartheta_{1}\left(z-w+U_{2}\right)}{\vartheta_{1}\left(U_{2}\right)} \frac{\vartheta_{1}\left(z-w+Y_{2}-U_{2}\right)}{\vartheta_{1}\left(Y_{2}-U_{2}\right)}
\end{aligned}
$$

Note that $g_{s}(z, w), P(z, w)$ is doubly periodic and $g_{s}$ has a double pole at $z=w$ with coefficient $1 . U_{1}, U_{2}$ are determined by the requirement that the single pole is absent in $g_{s}$ when $z \rightarrow w$. After some simple algebra it is easy see from the construction of $P(z, w)$ that this requirement is satisfied if $F_{1}$ and $F_{2}$ obey the conditions

$$
\left.\partial_{z} F_{1}(z, w)\right|_{z=w}=0,\left.\quad \partial_{z} F_{2}(z, w)\right|_{z=w}=0 .
$$


This leads to the equations

$$
\begin{aligned}
& \frac{\vartheta_{1}^{\prime}\left(U_{1}\right)}{\vartheta_{1}\left(U_{1}\right)}+\frac{\vartheta_{1}^{\prime}\left(Y_{1}-U_{1}\right)}{\vartheta_{1}\left(Y_{1}-U_{1}\right)}=0, \\
& \frac{\vartheta_{1}^{\prime}\left(U_{2}\right)}{\vartheta_{1}\left(U_{2}\right)}+\frac{\vartheta_{1}^{\prime}\left(Y_{2}-U_{2}\right)}{\vartheta_{1}\left(Y_{2}-U_{2}\right)}=0 .
\end{aligned}
$$

Note that 1.h.s. of each equation is a doubly periodic meromorphic function of $U_{1}, U_{2}$ respectively. There is a simple pole at $U_{1}=0$ and $Y_{1}-U_{1}=0$ for the first 1.h.s. of the first equation. Similarly l.h.s. of the second equation has a simple pole at $U_{2}=0$ and $Y_{2}-U_{2}=0$. Therefore each of these functions has two zeros say at $U_{1}^{(0)}$ and $Y_{1}-U_{1}^{(0)}$ and similarly for the second function. We will choose $U_{1}=U_{1}^{(0)}$ and $U_{2}=U_{2}^{(0)}$. Then the equations in (2.32) are satisfied identically. We will not need the explicit solution $U_{i}^{(0)}$ or even the explicit form of $P(z, w)$ for evaluating the quantum part of the two point function. From the constraints on $P(z, w)$ imposed by the fact that $g_{s}(z, w)$ has a double pole with coefficient 1 and no single pole, it is easy to see that $P(z, w)$ obeys the following equation

$$
\begin{aligned}
& \gamma_{1-k / n}(w) \gamma_{k / n}(w)\left(\left.\frac{\partial}{\partial z} \frac{\partial}{\partial w} P(z, w)\right|_{z=w}+\left.\frac{\partial^{2}}{\partial z^{2}} P(z, w)\right|_{z=w}\right) \\
& =\left(1-\frac{k}{n}\right) \frac{\vartheta_{1}^{\prime \prime}\left(w-z_{1}\right)}{\vartheta_{1}\left(w-z_{1}\right)}+\frac{k}{n} \frac{\vartheta_{1}^{\prime \prime}\left(w-z_{2}\right)}{\vartheta_{1}\left(w-z_{2}\right)}+\frac{\vartheta_{1}^{\prime}\left(w-z_{1}\right) \vartheta_{1}^{\prime}\left(w-z_{2}\right)}{\vartheta_{1}\left(w-z_{1}\right) \vartheta_{1}\left(w-z_{2}\right)} .
\end{aligned}
$$

We now determined $A, B$ from the monodromy conditions given in (2.11). We define the following integrals along the two cycles of the torus.

$$
\begin{array}{ll}
W_{1}^{1}=\int_{\gamma^{1}} d z \omega_{1}(z), & W_{1}^{2}=\int_{\gamma^{1}} d \bar{z} \bar{\omega}_{2}(\bar{z}), \\
W_{2}^{1}=\int_{\gamma^{2}} d z \omega_{1}(z), & W_{2}^{2}=\int_{\gamma^{2}} d \bar{z}^{2} \bar{\omega}_{2}(\bar{z}) .
\end{array}
$$

Then the equations (2.11) reduce to

$$
\begin{aligned}
& A \omega_{2}(w) W_{1}^{1}+B \omega_{2}(w) W_{1}^{2}=-\int_{\gamma^{1}} d z g_{s}(z, w), \\
& A \omega_{2}(\omega) W_{2}^{1}+B \omega_{2}(w) W_{2}^{2}=-\int_{\gamma^{2}} d z g_{s}(z, w) .
\end{aligned}
$$

The solution for $A, B$ are given by

$$
\begin{aligned}
A \omega_{2}(w) & =-\frac{1}{|W|}\left(W_{2}^{2} \int_{\gamma^{1}} d z g_{s}(z, w)-W_{1}^{2} \int_{\gamma^{2}} d z g_{s}(z, w)\right), \\
B \omega_{2}(w) & =-\frac{1}{|W|}\left(W_{1}^{1} \int_{\gamma^{2}} d z g_{s}(z, w)-W_{2}^{1} \int_{\gamma^{1}} d z g_{s}(z, w)\right), \\
|W| & =W_{1}^{1} W_{2}^{2}-W_{2}^{1} W_{1}^{2} .
\end{aligned}
$$

Note that these are just functions of $w$. Therefore the Greens' functions are

$$
\begin{aligned}
& g(z, w)=g_{s}(z, w)-\omega_{1}(z) \frac{1}{|W|}\left(W_{2}^{2} \int_{\gamma^{1}} d y g_{s}(y, w)-W_{1}^{2} \int_{\gamma^{2}} d y g_{s}(y, w)\right), \\
& h(\bar{z}, w)=-\bar{\omega}_{2}(\bar{z}) \frac{1}{|W|}\left(W_{1}^{1} \int_{\gamma^{2}} d y g_{s}(y, w)-W_{2}^{1} \int_{\gamma^{1}} d y g_{s}(y, w)\right) .
\end{aligned}
$$


We proceed to the second stage and obtain the stress tensor. Taking the limit given in (2.13) and using the identity (2.33) we obtain

$$
\begin{aligned}
\langle T(z)\rangle= & \frac{1}{2}\left[\frac{k}{n} \frac{\vartheta_{1}^{\prime}\left(z-z_{1}\right)}{\vartheta_{1}^{\prime}\left(z-z_{1}\right)}+\left(1-\frac{k}{n}\right) \frac{\vartheta_{1}^{\prime}\left(z-z_{2}\right)}{\vartheta_{1}^{\prime}\left(z-z_{2}\right)}\right]\left[\left(1-\frac{k}{n}\right) \frac{\vartheta_{1}^{\prime}\left(z-z_{1}\right)}{\vartheta_{1}\left(z-z_{1}\right)}+\frac{k}{n} \frac{\vartheta_{1}^{\prime}\left(z-z_{2}\right)}{\vartheta_{1}^{\prime}\left(z-z_{2}\right)}\right] \\
& -\left.\frac{1}{2} \gamma_{1-\frac{k}{n}}(z) \gamma_{k}(z) \frac{\partial}{\partial z} \frac{\partial}{\partial w} P(z, w)\right|_{w=z}-\frac{1}{3} \frac{\vartheta_{1}^{\prime \prime \prime}(0)}{\vartheta_{1}^{\prime}(0)} \\
& -\omega_{1}(z) \frac{1}{|W|}\left(W_{2}^{2} \int_{\gamma^{1}} d y g_{s}(y, z)-W_{1}^{2} \int_{\gamma^{2}} d y g_{s}(y, z)\right) .
\end{aligned}
$$

We now obtain differential equations for the quantum part of the two point function using the identity in (2.15). A similar set of differential equations can be obtained by considering the expectation value of the anti-holomorphic stress tensor in the presence of the twist fields. We will examine the differential equation for $\partial_{z_{1}} \ln Z_{k, n}^{q u}$. Taking the limit in (2.15) we obtain

$$
\begin{aligned}
\partial_{z_{1}} \ln Z_{k, n}^{q u}= & \frac{1}{2}\left(\left(\frac{k}{n}\right)^{2}+\left(1-\frac{k}{n}\right)^{2}\right) \frac{\theta_{1}^{\prime}\left(z_{1}-z_{2}\right)}{\theta_{1}\left(z_{1}-z_{2}\right)} \\
& -\left.\frac{1}{2 \theta_{1}^{\prime}(0)} \frac{1}{\vartheta_{1}\left(z_{1}-z_{2}\right)} \frac{\partial}{\partial z} \frac{\partial}{\partial w} P(z, w)\right|_{z=w=z_{1}} \\
& -\frac{1}{|W|}\left(W_{2}^{2} \int_{\gamma^{1}} d z \omega_{1}(z) \Lambda(z)-W_{1}^{2} \int_{\gamma^{2}} d z \omega_{1}(z) \Lambda(z)\right)
\end{aligned}
$$

where

$$
\Lambda(z)=\lim _{w \rightarrow z_{1}}\left(\frac{\omega_{1}(w)}{\gamma_{1-k / n}(w)}\right) \frac{\gamma_{1-k / n}(z)}{\omega_{1}(z)} \frac{\vartheta_{1}^{\prime}(0) P\left(z, z_{1}\right)}{\vartheta_{1}^{2}\left(z-z_{1}\right) \vartheta_{1}\left(z_{1}-z_{2}\right)}
$$

We will now rewrite $\Lambda$ in terms of the cut differential $\omega_{1}$. To do this we use the form of $P(z, w)$ given in (2.30) and the equations (2.32) to Taylor expand $P\left(z, z_{1}\right)$ about $z_{1}$ and obtain

$$
\lim _{z \rightarrow z_{1}} \Lambda(z) \sim \frac{1-k / n}{z-z_{1}}
$$

It is easy to see that this is also the leading singularity of the function $f(z)=$ $\omega_{1}(z)^{-1} \partial_{z_{1}} \omega_{1}(z)$. The functions $\Lambda(z)$ and $f(z)$ are meromorphic doubly periodic functions which agree at their poles. Therefore their difference must be a constant. Thus we have the equation

$$
\omega_{1}(z) \Lambda(z)=\partial_{z_{1}} \omega_{1}+S \omega_{1}(z)
$$

To obtain $S$, we examine the behaviour of the of $\Lambda(z)-\left(\omega_{1}(z)\right)^{-1} \partial_{z_{1}} \omega_{1}(z)$ as $z \rightarrow z_{1}$. This results in

$$
S=\frac{\left.\partial_{z}^{2} P\left(z, z_{1}\right)\right|_{z=z_{1}}}{2 \vartheta_{1}^{\prime}(0) \vartheta_{1}\left(z_{1}-z_{2}\right)}
$$

We now substitute (2.42) and use the identity (2.33) to write the differential equation for the two point correlator as

$$
\partial_{z_{1}} Z_{k, n}^{q u}=-\frac{1}{|W|}\left(W_{2}^{2} \partial_{z_{1}} W_{1}^{1}-W_{2}^{2} \partial_{z_{1}} W_{2}^{1}\right)+\partial_{z_{1}} \ln \left[\vartheta_{1}\left(z_{1}-z_{2}\right)^{-(1-k / n) k / n}\right] .
$$


Note that the first term can be rewritten as

$$
-\frac{1}{|W|}\left(W_{2}^{2} \partial_{z_{1}} W_{1}^{1}-W_{2}^{2} \partial_{z_{1}} W_{2}^{1}\right)=-\partial_{z_{1}} \ln |W| .
$$

This is because the integrals $W_{2}^{2}, W_{2}^{1}$ depend on anti-holomorphic coordinates. Using this input, and integrating the differential equation we obtain

$$
Z_{k, n}^{q u}=f\left(\tau, k / n, z_{2}, \bar{z}_{1}, \bar{z}_{2}\right)|W|^{-1}\left(\vartheta_{1}\left(z_{1}-z_{2}\right)^{-(1-k / n) k / n}\right) .
$$

One can use the similar procedure to obtain a differential equation for $z_{2}$ and also for the anti-holomorphic coordinates $\bar{z}_{1}, \bar{z}_{2}$ by considering the expectation value of the the anti-holomorphic stress tensor $\langle\bar{T}(\bar{z})\rangle$. This leads to

$$
Z_{k, n}^{q u}=f(\tau, k / n)|W|^{-1} \vartheta_{1}\left(z_{1}-z_{2}\right)^{-(1-k / n) k / n}{\overline{\vartheta_{1}\left(z_{1}-z_{2}\right)}}^{-(1-k / n) k / n} .
$$

The normalization $f$ can be determined by demanding that in the limit $z_{1} \rightarrow z_{2}$, the two point function is normalized to one. It is easy to see that in this limit the integrals reduce to

$$
\begin{array}{ll}
W_{1}^{1} \rightarrow \int_{0}^{1} d z=1, \quad W_{2}^{2} \rightarrow \int_{0}^{\tau} d \bar{z}=\tau, \\
W_{2}^{1} \rightarrow \int_{0}^{\bar{\tau}} d z=\bar{\tau}, \quad W_{1}^{2} \rightarrow \int_{0}^{1} d \bar{z}=1 .
\end{array}
$$

Note that we are taking the cycle $\gamma_{2}$ to be such that $\bar{z}$ runs from 0 to $\tau$, which implies $z$ runs from 0 to $\bar{\tau}$. Therefore $|W| \rightarrow 2 i \tau_{2}$ where $\tau_{2}$ is the imaginary part of the modulus of the torus. Using this argument we have

$$
Z_{k, n}^{q u}=\frac{2 i \tau_{2}}{|W|}\left(\frac{\vartheta_{1}^{\prime}(0)}{\vartheta_{1}\left(z_{1}-z_{2}\right)}\right)^{(1-k / n) k / n}\left(\overline{\frac{\vartheta_{1}^{\prime}(0)}{\vartheta_{1}\left(z_{1}-z_{2}\right)}}\right)^{(1-k / n) k / n} .
$$

The quantum contribution of the Ashkin-Teller model [19] can be recovered from the above expression by setting $k / n=1 / 2$.

\subsection{Classical contribution}

In this sub-section we will evaluate the classical contribution due to the various saddle points of the action. These saddle points exist when the complex boson is compactified on a torus. Consider the torus to be a square torus of radius $\mathcal{R}+i \mathcal{R}$. Then the twisted sector boundary conditions for classical solutions is of the form

$$
X_{c l}\left(e^{2 \pi i} z, e^{-2 \pi i} \bar{z}\right)=e^{2 \pi i k / n} X_{c l}(z, \bar{z})+v,
$$

where $v$ is a vector related to translations in the lattice $\Lambda_{\underline{k}}$ which will be specified precisely subsequently. These boundary conditions give rise to the following monodromy conditions.

$$
\begin{aligned}
& \oint_{\gamma_{a}} d z \partial_{z} X_{c l}+\oint_{\gamma_{a}} d \bar{z} \partial_{\bar{z}} X_{c l}=v_{a} \quad(a=1,2) \\
& \oint_{\gamma_{a}} d z \partial_{z} \bar{X}_{c l}+\oint_{\gamma_{a}} d \bar{z} \partial_{\bar{z}} \bar{X}_{c l}=\bar{v}_{a} .
\end{aligned}
$$


The subscript $a$ denotes the two cycles of the worldsheet torus. The classical action is given by

$$
S_{c l}[X, \bar{X}]=\frac{1}{4 \pi} \int_{D} d^{2} z\left(\partial_{z} X_{c l} \partial_{\bar{z}} \bar{X}_{c l}+\partial_{\bar{z}} X_{c l} \partial_{z} \bar{X}_{c l}\right)
$$

We now have to evaluate the classical action given below for solutions satisfying the boundary conditions in (2.51). The domain of integration $D$ will be specified below. The classical solutions to the equations of motion can also be written in terms of the cut differentials. They are given by

$$
\begin{aligned}
\partial_{z} X_{c l}(z) & =a \omega_{1}(z), & \partial_{\bar{z}} X_{c l}(\bar{z}) & =b \bar{\omega}_{2}(\bar{z}), \\
\partial_{z} \bar{X}_{c l}(z) & =\tilde{a} \omega_{2}(z), & \partial_{\bar{z}} \bar{X}_{c l}(\bar{z}) & =\tilde{b} \bar{\omega}_{1}(\bar{z}) .
\end{aligned}
$$

Note that these solutions satisfy the required holomorphic/anti-holomorphic conditions and the singularity properties near the twist operators. Substituting (2.53) in (2.51) we get the following system of equations.

$$
\left(\begin{array}{ll}
W_{1}^{1} & W_{1}^{2} \\
W_{1}^{2} & W_{2}^{2}
\end{array}\right)\left(\begin{array}{l}
a \\
b
\end{array}\right)=\left(\begin{array}{l}
v_{1} \\
v_{2}
\end{array}\right), \quad\left(\begin{array}{ll}
\bar{W}_{1}^{2} & \bar{W}_{1}^{1} \\
\bar{W}_{2}^{2} & \bar{W}_{2}^{1}
\end{array}\right)\left(\begin{array}{c}
\tilde{a} \\
\tilde{b}
\end{array}\right)=\left(\begin{array}{c}
\bar{v}_{1} \\
\bar{v}_{2}
\end{array}\right) .
$$

We have defined the integrals of the cut differentials on the cycles of the worldsheet torus in (2.34). The solutions for $a, b, \tilde{a}, \tilde{b}$ are as follows

$$
\begin{array}{ll}
a=\frac{W_{2}^{2} v_{1}-W_{1}^{2} v_{2}}{|W|}, & b=\frac{-W_{2}^{1} v_{1}+W_{1}^{1} v_{2}}{|W|}, \\
\tilde{a}=\frac{\bar{W}_{1}^{1} \bar{v}_{2}-\bar{W}_{2}^{1} \bar{v}_{1}}{|\bar{W}|}, & \tilde{b}=\frac{\bar{W}_{2}^{2} \bar{v}_{1}-\bar{W}_{1}^{2} \bar{v}_{2}}{|\bar{W}|} .
\end{array}
$$

Substituting these in the solutions (2.53) and then evaluating the action (2.52) we encounter the following area integrals.

$$
I_{1}=\int d^{2} z\left|\omega_{1}\right|^{2}, \quad I_{2}=\int d^{2} z\left|\omega_{2}\right|^{2} .
$$

To perform these integrals we use the method developed in [19] and [18]. Let us focus on the integral $I_{1}$. The area integral is carried out over the shaded domain $d$ shown in figure 1 . In this domain, there exists an analytic function $f(z)$

$$
f(z)=\int_{z_{0}}^{z} d t \omega_{1}(z)
$$

such that $d f(z)=\omega_{1}(z)$. Substituting this in the expression for $I_{1}$ we obtain

$$
I_{1}=\frac{1}{2 i} \int d z \wedge \bar{d} z \frac{\partial}{\partial z}\left(f(z) \bar{\omega}_{1}(\bar{z})\right) .
$$

We can now use Green's theorem to write the area integral as an integral over the contour whose boundary is the usual parallelogram representing the torus along with the thin 


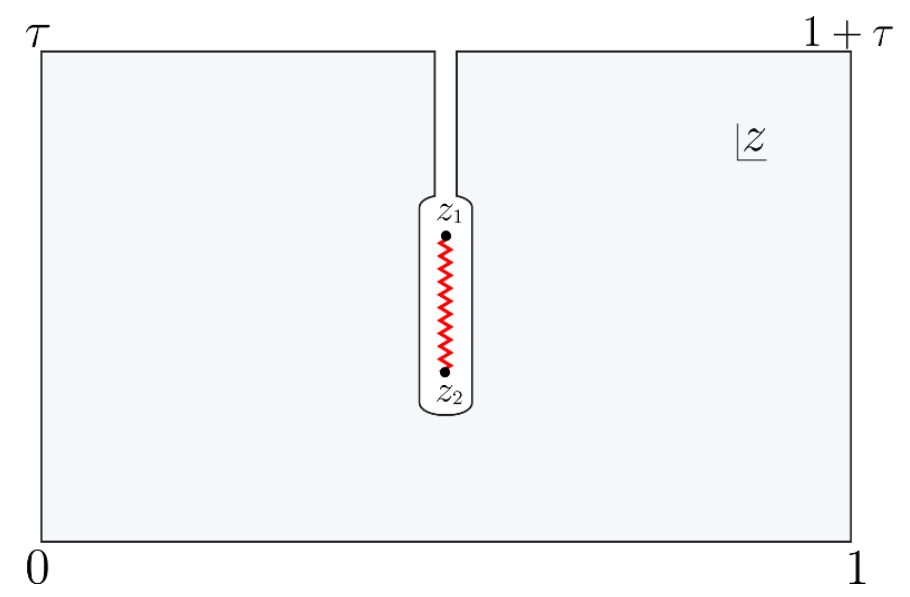

Figure 1. Simply connected domain of the area integral.

neck as shown in the figure 1 . The integral along the cuts vanishes. This is because the combination $f(z) \bar{\omega}_{1}(z)$ does not jump across the cut. The simplest way to see this is to take $z_{0}=z_{1}$ and then one sees that the monodromy of $\omega_{1}(z)$ cancels that of $\bar{\omega}_{1}(z)$. Thus the integral $I_{1}$ can be written as

$$
I_{1}=\frac{1}{2 i}\left(\bar{W}_{1}^{1} W_{2}^{1}-W_{1}^{1} \bar{W}_{2}^{1}\right)
$$

With a similar analysis we can show that

$$
I_{2}=\frac{1}{2 i}\left(W_{1}^{2} \bar{W}_{2}^{2}-\bar{W}_{1}^{2} W_{2}^{2}\right)
$$

Now substituting these results in the classical action we obtain

$$
\begin{aligned}
S_{c l}= & \frac{1}{4 \pi \operatorname{det}(W) \operatorname{det}(\bar{W})}\left(\left|v_{1}\right|^{2} \operatorname{Im}\left(W_{2}^{2} W_{2}^{1} \operatorname{det}(\bar{W})\right)+\left|v_{2}\right|^{2} \operatorname{Im}\left(W_{1}^{2} W_{1}^{1} \operatorname{det}(\bar{W})\right)\right. \\
& \left.-\left(v_{1} \bar{v}_{2}+\bar{v}_{1} v_{2}\right) \operatorname{Im}\left(W_{2}^{1} W_{1}^{2} \operatorname{det}(\bar{W})\right)\right)
\end{aligned}
$$

We will further simplify the classical action as well as the quantum contribution $Z_{k, n}^{q u}$ after we examine the integrals of the cut differentials.

\subsection{Integrals of the cut differentials}

There are four integrals of the cut differentials along the two cycles of the worldsheet torus. To simplify the integrals further we choose the points of insertions of the twist and the anti-twist operators to be at $z_{1}=y$ and $z_{2}=1-y$ (where $0 \leq y \leq \frac{1}{2}$ ) as shown in figure 2 . We will also choose the modular parameter of the worldsheet torus to be purely imaginary, that is

$$
\tau=i \beta .
$$




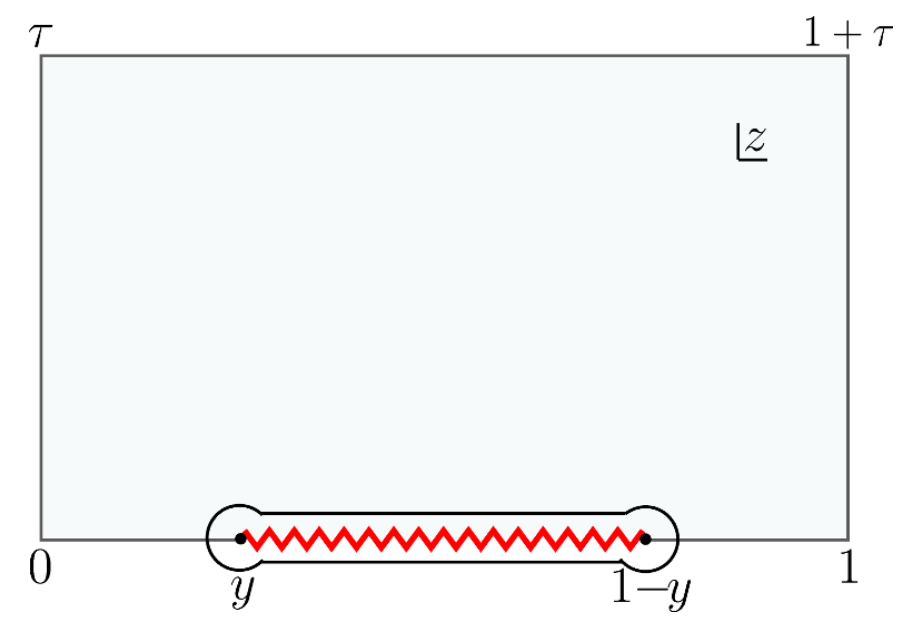

Figure 2. Fundamental domain of the torus lattice showing the location of the interval.

From now onwards in this paper we will work with these choices. Then the following properties of these integrals can be shown ${ }^{5}$

$$
\left(W_{1}^{1}\right)^{*}=W_{1}^{2}, \quad\left(W_{1}^{1}\right)^{*}=W_{1}^{1}, \quad\left(W_{2}^{1}\right)^{*}=-W_{2}^{1}, \quad\left(W_{2}^{1}\right)^{*}=W_{2}^{2} .
$$

These identities imply that

- $W_{1}^{1}$ and $W_{1}^{2}$ are purely real.

- $W_{2}^{1}$ and $W_{2}^{2}$ are purely imaginary.

- $\operatorname{det}(W)$ and $\operatorname{det}(\bar{W})$ are purely imaginary. $\operatorname{det}(W)=2 i \operatorname{Im}\left(W_{1}^{1} W_{2}^{2}\right)$.

Thus it is sufficient to examine the integrals $W_{1}^{1}$ and $W_{2}^{2}$. An important result which emerges during the proof of these relations is that the integral along the cut vanishes

$$
\int_{y}^{1-y} d z \omega_{1}(z)=0
$$

Thus the contribution of the $W_{1}^{1}$ integral arises only from the intervals $[0, y]$ and $[1-y, 1]$. Therefore we can write

$$
W_{1}^{1}=\int_{0}^{y} d z \omega_{1}(z)+\int_{1-y}^{y} d z \omega_{1}(z)
$$

Let us define

$$
L=1-2 y,
$$

the separation between the two insertions of the twist operator. We will also keep track of the dependence of the integrals on the interval $L$ and the modular parameter of the worldsheet torus $\tau$. The integrals along the two cycles are related by the following equation

$$
W_{2}^{2}(L, \tau)=\tau W_{1}^{1}(-L, \tau) .
$$

\footnotetext{
${ }^{5}$ See appendix B for the proofs.
} 
It can be seen from term by term expansion of $W_{1}^{1}$ that

$$
W_{1}^{1}(L, \tau)=W_{1}^{1}(-L, \tau)
$$

Therefore we have

$$
W_{2}^{2}(L, \tau)=\tau W_{1}^{1}(L, \tau) .
$$

Using modular transformation we can obtain the high temperature expansions of the integral $W_{2}^{2}$ from the relation

$$
W_{2}^{2}(L, \tau)=\tau e^{\frac{i \pi L^{2}}{\tau} \frac{k}{n}\left(1-\frac{k}{n}\right)} W_{1}^{1}(L / \tau,-1 / \tau) .
$$

Once the high temperature expansion of $W_{2}^{2}$ is known, the high temperature expansion of $W_{1}^{1}$ can be obtained by the relation (2.69). All these relations are systematically proved in appendix B. The high-temperature expansion of $W_{2}^{2}$ is derived in appendix $\mathrm{B}$ and is given by

$$
\begin{aligned}
& W_{2}^{2}(L, \tau)_{k, n} \\
& =i e^{\frac{\pi}{\beta} \frac{k}{n}\left(1-\frac{k}{n}\right) L^{2}} \beta \\
& \times\left[1-\frac{2}{n^{2}}\left(-k^{2}+k n \cosh \left(\frac{2 \pi L(n-k)}{n \beta}\right)+(k-n)\left(k \cosh \left(\frac{2 \pi L}{\beta}\right)-n \cosh \left(\frac{2 \pi k L}{n \beta}\right)\right)+k n-n^{2}\right) q^{2}\right. \\
& +\frac{1}{2 n^{4}}\left(2 n \left(3(k-n)\left(k^{2}-k n+2 n^{2}\right) \cosh \left(\frac{2 \pi k L}{n}\right)\right.\right. \\
& \quad+k\left(-3\left(k^{2}-k n+2 n^{2}\right) \cosh \left(\frac{2 \pi L(n-k)}{n}\right)-(k-2 n)(k-n) \cosh \left(\frac{2 \pi L(k+n)}{n}\right)\right. \\
& \left.\left.\quad+(k-n)(k+n) \cosh \left(\frac{2 \pi L(k-2 n)}{n}\right)\right)\right) \\
& \quad-4 k(k-n)\left(k^{2}-k n+4 n^{2}\right) \cosh \left(\frac{2 \pi L}{\beta}\right)+3\left(k^{4}-2 k^{3} n+7 k^{2} n^{2}-6 k n^{3}+4 n^{4}\right) \\
& \left.\left.+k(k-n)(k+n)(k-2 n) \cosh \left(\frac{4 \pi L}{\beta}\right)\right) q^{4}+\mathcal{O}\left(q^{6}\right)\right] .
\end{aligned}
$$

The high temperature expansion of $W_{1}^{1}$ can then be obtained by the relation in (2.69).

\subsection{Rényi entropies of a compact boson}

We are now ready to put the results together to obtain the Rényi entropies for a compact boson. The classical action in (2.61) simplifies on using the reality conditions to the following

$$
S_{c l}=\frac{1}{4 \pi \operatorname{Im}(\operatorname{det}(W))}\left(\left|v_{1} W_{2}^{2}\right|^{2}+\left|v_{2} W_{1}^{1}\right|^{2}\right)
$$

We can now use the relation between $W_{1}^{1}$ and $W_{2}^{2}$ given in (2.69) to reduce the classical action to

$$
S_{c l}=\frac{1}{8 \pi \tau_{2}}\left(\left|v_{1}\right|^{2}|\tau|^{2}+\left|v_{2}\right|^{2}\right) .
$$

Thus $S_{c l}$ is independent of the interval. This simplification is the result of the choice of the interval which is along one cycle of the torus as well as the fact that the spacetime torus 
is square and the worldsheet torus is rectangular. The lattice translations $v_{1}$ and $v_{2}$ are given as follows $[10,17]$

$$
v_{j}=\left(1-e^{\frac{2 \pi i k}{n}}\right) \xi_{j},
$$

and $\xi_{j}$ is a general lattice vector in $\Lambda_{\frac{k}{n}}$. This lattice is defined as follows

$$
\Lambda_{\frac{k}{n}} \equiv\left\{q=\mathcal{R} \sum_{j=0}^{n-1} e^{\frac{2 \pi i k j}{n}}\left(m_{j, 1}+i m_{j, 2}\right) ; m_{j, 1}, m_{j, 2} \in \mathbf{Z}\right\}
$$

Note that we are working with a square torus of radius $\mathcal{R}$ and this lattice results because of the Fourier transformation involved from the replica fields to the eigenbasis in which the shifts between replicas are represented by multiplication the phases $e^{\frac{2 \pi i k}{n}}$ as given in (2.2). Thus we can write

$$
\xi_{p}=\mathcal{R} \sum_{j=0}^{n-1} e^{\frac{2 \pi i k j}{n}}\left(m_{j, 1}^{(p)}+i m_{j, 2}^{(p)}\right) .
$$

The superscript $(p)$ labels the two cycles of the worldsheet torus directions along which the monodromies are evaluated. The rest of the analysis proceeds similar to the analysis done in [10]. We have

$$
\left|\xi_{p}\right|^{2}=\mathcal{R}^{2}\left[\sum_{q=1,2}\left[m_{q}^{(p)}\right]^{t} \cdot C_{k / n} \cdot m_{q}^{(p)}+\sum_{r, s=0}^{n-1}\left(m_{r, 1}^{(p)} m_{s, 2}^{(p)}-m_{s, 1}^{(p)} m_{r, 2}^{(p)}\right)\left(S_{k / n}\right)_{r s}\right] .
$$

Here, $m_{q}^{(p)} \in \mathbf{Z}$ and

$$
\left(C_{k / n}\right)_{r s} \equiv \cos \left[2 \pi \frac{k}{n}(r-s)\right], \quad\left(S_{k / n}\right)_{r s} \equiv\left[2 \pi \frac{k}{n}(r-s)\right] .
$$

The classical action (2.73) can be written in terms of $\xi_{p}$ as

$$
S_{c l}=\frac{\sin ^{2}\left(\frac{\pi k}{n}\right)}{2 \pi \tau_{2}}\left(\left|\xi_{1}\right|^{2}|\tau|^{2}+\left|\xi_{2}\right|^{2}\right) .
$$

Thus the classical configurations are labelled by the integers $m_{j}^{(p)}$ and we have to sum over these classical configurations. The contribution of the sum over classical configurations to the two point functions of the twist operator is therefore given by

$$
\begin{aligned}
Z_{c l} & =\prod_{k=0}^{n-1} Z_{k, n}^{c l} \\
& =\left[\sum_{m \in \mathbf{Z}^{n}} \prod_{k=0}^{n-1} \exp \left\{-\frac{\left(1+|\tau|^{2}\right) \sin ^{2}\left(\frac{\pi k}{n}\right)}{2 \pi \tau_{2}} m^{t} \cdot C_{k / n} \cdot m\right\}\right]^{2}, \\
& =\left[\sum_{m \in \mathbf{Z}^{n}} \exp \left\{i \pi\left[m^{t} \cdot \Omega \cdot m\right]\right\}\right]^{2}
\end{aligned}
$$


where we have defined

$$
\Omega_{r s}=\frac{i\left(1+|\tau|^{2}\right) \mathcal{R}^{2}}{2 \pi \tau_{2}} \sum_{k=0}^{n-1} \sin ^{2}\left(\frac{\pi k}{n}\right) \cos \left(\frac{2 \pi k}{n}(r-s)\right),
$$

where the indices $r$ and $s$ run over $1,2, \cdots, n$. The sum in (2.80) is however divergent since $\Omega$ has a zero eigenvalue. Following [10], we can absorb this divergence in the normalization factor. Essentially this leads to fact that $r, s$ run from $1,2, \cdots n-1$. Thus the partition function can be written in terms of the Riemann-Siegel function as

$$
Z_{c l}=[\Theta(0 \mid \eta \Gamma)]^{2} .
$$

Here

$$
\Theta(0 \mid \eta \Gamma)=\sum_{m \in \mathbf{Z}^{n-1}} \exp \left(i \pi m^{t} \cdot \Gamma \cdot m\right), \quad \eta=\frac{i}{2} \frac{n\left(1+|\tau|^{2}\right)}{2 \pi \tau_{2}} \mathcal{R}^{2}
$$

and

$$
\begin{aligned}
\Gamma_{r s} & =\frac{4}{n} \sum_{k=1}^{n-1} \sin ^{2}\left(\frac{\pi k}{n}\right) \cos \left(\frac{2 \pi k}{n}(r-s)\right) \\
& =2\left(\delta_{r, s}-\frac{1}{2} \delta_{r, s+1}-\frac{1}{2} \delta_{r, s-1}\right) .
\end{aligned}
$$

and $r, s$ run from $1,2, \cdots n-1$. The above sparse matrix corresponds the Cartan matrix of $\mathrm{SU}(n)$. The Riemann-Siegel function is then the partition function of the $A_{n-1}$ root lattice. This can be conveniently expressed as the $\vartheta_{3}$-series [32]. The Riemann-Siegel function for the matrix $\Gamma$ given by (2.84) is thus given by

$$
\Theta(0 \mid \eta \Gamma)=\frac{\sum_{k=0}^{n-2} \vartheta_{3}\left(\frac{k}{n-1} \mid \eta\right)^{n-1}}{(n-1) \vartheta_{3}(0 \mid(n-1) \eta)} .
$$

The classical part (2.82) upto a normalization reduces to

$$
Z_{c l}=\left[\frac{\sum_{k=0}^{n-2} \vartheta_{3}\left(\frac{k}{n-1} \mid \eta\right)^{n-1}}{(n-1) \vartheta_{3}(0 \mid(n-1) \eta)}\right]^{2} .
$$

It can be easily seen from the above that in the decompactification regime $\mathcal{R} \rightarrow \infty, Z_{c l}=1$. The fact that we obtain the Riemann-Siegel theta function corresponding to the $A_{n-1}$ root lattice is a result of our special choice of the moduli. We have chosen a rectangular worldsheet torus as well as a square spacetime lattice. Furthermore the cut - that is the location of the twist operators - is along the spatial cycle of the torus. Due to these choices we obtained several simplifications of the integrals of the cut differentials which lead to the Riemann-Siegel theta function associated with $A_{n-1}$. For a generic choice of the torus moduli or the location of the twist operators we do not expect this simplification. 


\section{The full partition function}

We can now write the full partition function taking contributions from the quantum (2.49) and classical parts (2.82)

$$
\begin{gathered}
Z[n]=\left(\prod_{k=0}^{n-1} 2 i \tau_{2}|W(k, n)|^{-1}\left(\frac{\vartheta_{1}\left(z_{2}-z_{1} \mid \tau\right)}{\vartheta_{1}^{\prime}(0 \mid \tau)} \frac{\overline{\vartheta_{1}\left(z_{2}-z_{1} \mid \tau\right)}}{\overline{\vartheta_{1}^{\prime}(0 \mid \tau)}}\right)^{-\frac{k}{n}\left(1-\frac{k}{n}\right)}\right) \\
\times\left[\frac{\sum_{k=0}^{n-2} \vartheta_{3}\left(\frac{k}{n-1} \mid \eta\right)^{n-1}}{(n-1) \vartheta_{3}(0 \mid(n-1) \eta)}\right]^{2} .
\end{gathered}
$$

The Rényi entropy can be derived from the above by using

$$
S_{n}=\frac{1}{1-n}(\log Z[n]-n \log Z[1]) .
$$

Here, $Z[1]$ is the CFT partition function on the original spacetime (i.e. a torus in our case) and $Z[n]$ is the partition function on the $\mathbb{Z}_{n}$ orbifolded cover. Using the above expression for $Z[n]$ (and $W(0,1)=2 \tau$ for any $z_{2}-z_{1}$, which is shown in (B.6)), we can evaluate the Rényi entropy to be

$$
\begin{gathered}
S_{n}=\frac{1}{1-n} \log \left|\left(\prod_{k=0}^{n-1}\left|\frac{W(k, n)}{2 \tau}\right|^{-1}\left(\frac{\vartheta_{1}\left(z_{2}-z_{1}\right)}{\vartheta_{1}^{\prime}(0)} \frac{\overline{\vartheta_{1}\left(z_{2}-z_{1}\right)}}{\overline{\vartheta_{1}^{\prime}(0)}}\right)^{-\frac{k}{n}\left(1-\frac{k}{n}\right)}\right)\right| \\
+\frac{1}{1-n} \log \left|\left[\frac{\sum_{k=0}^{n-2} \vartheta_{3}\left(\frac{k}{n-1} \mid \eta\right)^{n-1}}{(n-1) \vartheta_{3}(0 \mid(n-1) \eta)}\right]^{2}\right| .
\end{gathered}
$$

We have thus obtained an exact analytical expression for the Rényi entropies of the free boson on the torus. It will be interesting to study this expression in detail and especially explore the dependence on the radius $\mathcal{R}$. Compact bosons are related to several $c=1$ statistical mechanical systems at criticality. Our result will be relevant to these systems.

\section{Rényi entropies for a non-compact boson}

For the rest of the paper we will study the Rényi entropies in the decompactification limit. In the $\mathcal{R} \rightarrow \infty$ only the quantum part contributes to the partition function. Therefore the full partition function (2.87) reduces to

$$
Z[n]=\left(\prod_{k=0}^{n-1}\left|\frac{W(k, n)}{2 \tau}\right|^{-1}\left(\frac{\vartheta_{1}\left(z_{2}-z_{1}\right)}{\vartheta_{1}^{\prime}(0)} \frac{\overline{\vartheta_{1}\left(z_{2}-z_{1}\right)}}{\overline{\vartheta_{1}^{\prime}(0)}}\right)^{-\frac{k}{n}\left(1-\frac{k}{n}\right)}\right) .
$$

The Rényi entropy (2.89) is this regime is

$$
S_{n}=-\frac{1}{1-n} \sum_{k=0}^{n-1} \frac{k}{n}\left(1-\frac{k}{n}\right) \log \left|\frac{\vartheta_{1}\left(z_{2}-z_{1}\right) \overline{\vartheta_{1}\left(z_{2}-z_{1}\right)}}{\vartheta_{1}^{\prime}(0)^{2}}\right|-\frac{1}{1-n} \sum_{k=0}^{n-1} \log \left|\frac{W(k, n)}{2 \tau}\right| .
$$



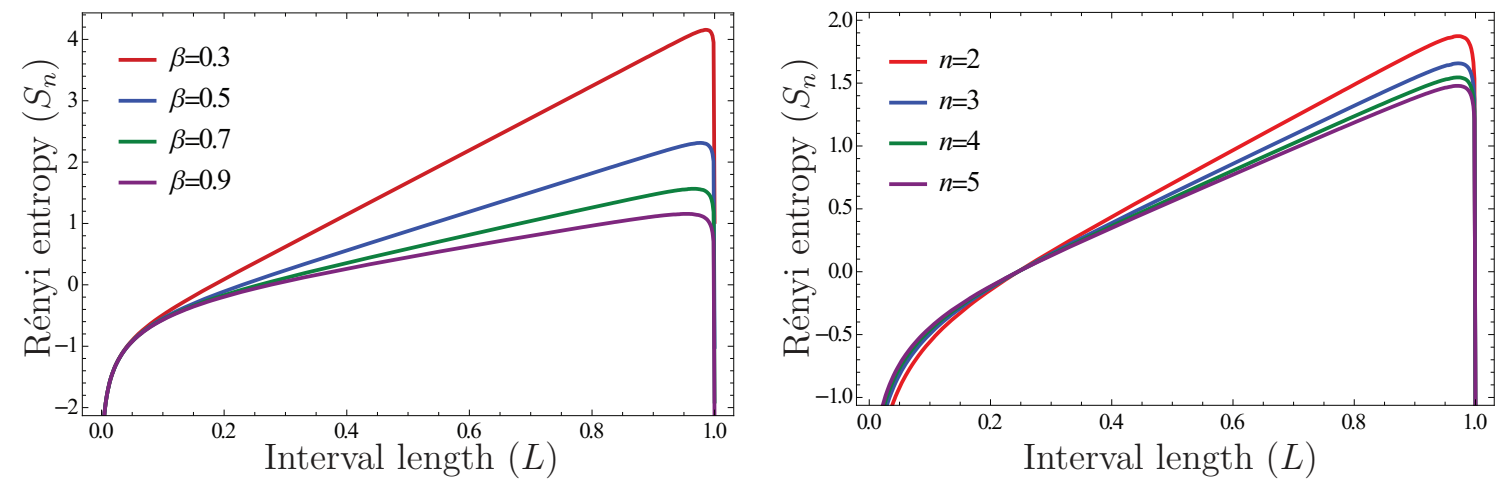

Figure 3. (Left) Plots of the $n=2$ Rényi entropies at $\beta=0.3,0.5,0.7$ and 0.9. (Right) Plots of the $n=2,3,4$ and 5 Rényi entropies at $\beta=0.6$.

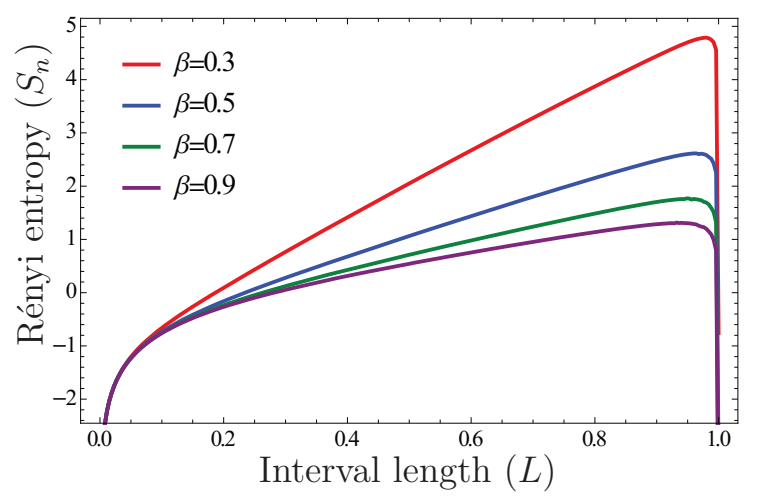

Figure 4. Plot of Rényi entropy extrapolated to $n \rightarrow 1$ at $\beta=0.3,0.5,0.7$ and 0.9 .

\subsection{Plots}

Although we have arrived at an expression for the Rényi entropy, the answer hides two integrals (B.2) involving $\vartheta$-functions. As shown in appendix B, these integrals can be performed in the high temperature or the low temperature expansion and analytical answers for the Rényi entropies can be obtained. However to get a picture of the behaviour of the Rényi entropy as the function of the interval size and the temperature it is useful to evaluate the integrals numerically and then plot the results.

In figure 3 we have plotted the $n=2$ Rényi entropies at four different temperatures. It can be seen that as the temperature increases the curve shifts higher. Also, the Rényi entropies for $n=2,3,4$ and 5 are plotted for $\beta=0.6$. These plots are similar to those obtained for the case of fermions on the torus $[14,15]$. We have also numerically extrapolated the answers for the Rényi entropies $(n=2,3, \cdots, 10)$ at four different temperatures to $n \rightarrow 1$ and obtained the entanglement entropy. The plot is shown the figure 4 . Note that for all these plots we have set the cut-off to unity.

As it can be seen the plots, the Rényi or entanglement entropy increases with rise in temperature. This can be reasoned as follows: increasing the temperature makes the system accessible to more excited states and thereby increases the Hilbert space we are tracing 
over. The peak at around $L=1$ is the result of the fact that at this point the entanglement entropy reduces to the thermal entropy which we now explore in the next subsection.

\subsection{Thermal entropy from entanglement entropy}

It is known that the entanglement entropy reduces to the thermal entropy when the size of the interval equals the length of the full spatial circle. Since there are no partial traces to perform, it is then given by the usual density matrix $\rho=\frac{e^{-\beta H}}{Z}$. The entanglement entropy is then

$$
\begin{aligned}
S_{E} & =\lim _{n \rightarrow 1} S_{n}^{(A)}=-\left.\frac{\partial}{\partial n} \log \left(\operatorname{Tr}\left(\rho^{n}\right)\right)\right|_{n=1} \\
& =-\left.\frac{\partial}{\partial n}\left(\log \left(\operatorname{Tr}\left(e^{-n \beta H}\right)\right)-n \log Z\right)\right|_{n=1} \\
& =\beta\langle H\rangle+\log Z=\beta(E-F)=S_{\text {thermal }},
\end{aligned}
$$

here ' $\operatorname{Tr}$ ' indicates not the partial trace but the trace over the full Hilbert space of states. In this section we shall calculate the entanglement entropy in this limit and verify that it does match with the thermal entropy. A similar check for the case of fermions on the torus was done in [14].

\section{Entanglement entropy in the limit $y \rightarrow 0$}

Let's define

$$
\begin{aligned}
S_{1} & =-\frac{1}{1-n} \sum_{k=0}^{n-1} \frac{k}{n}\left(1-\frac{k}{n}\right) \log \left|\frac{\vartheta_{1}\left(z_{2}-z_{1}\right) \overline{\vartheta_{1}\left(z_{2}-z_{1}\right)}}{\vartheta_{1}^{\prime}(0)^{2}}\right|, \\
& =\frac{n+1}{6 n} \log \left|\frac{\vartheta_{1}\left(z_{2}-z_{1}\right)}{\vartheta_{1}^{\prime}(0)^{2}}\right|^{2}, \\
S_{2} & =-\frac{1}{1-n} \sum_{k=0}^{n-1} \log \left|\frac{W(k, n)}{2 \tau}\right| .
\end{aligned}
$$

It follows from these definitions that $S_{n}=S_{1}+S_{2}$. We shall take the limit $y \rightarrow 0$ in which the length of the interval $(L=1-2 y)$ equals the size of the full spatial circle or the system size (see figure 2). We can then extract the finite part of the entanglement entropy as follows

$$
S_{\text {finite }}(L \rightarrow 1)=S(1-\epsilon)-S(\epsilon) .
$$

In this section, the interval length is denoted as $L$ and the full spatial circle $(R)$ is taken to be unity.

From (3.4) it can be seen that $S_{1}$ captures the universal piece of the entanglement entropy for $n \rightarrow 1$. The high-temperature expansion for $S_{1}$ can be obtained by performing 
a S-modular transformation $\tau \rightarrow-1 / \tau$ in equation (3.4)

$$
\begin{aligned}
\left.S_{1}(L)\right|_{n=1}= & \frac{2}{3} \log \left|\frac{\vartheta_{1}(L \mid \tau)}{2 \pi \eta(\tau)^{3}}\right|, \\
= & \frac{2}{3} \log \left|(-i \tau) e^{-\pi L^{2} / \beta} \frac{\vartheta_{1}\left(\frac{L}{\tau} \mid-\frac{1}{\tau}\right)}{2 \pi \eta\left(-\frac{1}{\tau}\right)^{3}}\right| \\
= & \frac{2}{3} \log \left|\frac{\beta}{2 \pi} \sinh \left(\frac{\pi L}{\beta}\right) e^{-\pi L^{2} / \beta}\right| \\
& +\frac{2}{3} \log \left|\prod_{m=1}^{\infty} \frac{\left(1-e^{-2 \pi m / \beta}\right)\left(1-e^{-2 \pi L m / \beta} e^{-2 \pi m / \beta}\right)\left(1-e^{2 \pi L m / \beta} e^{-2 \pi m / \beta}\right)}{\left(1-e^{-2 \pi m / \beta}\right)^{3}}\right| .
\end{aligned}
$$

In both the limits $L \rightarrow 1-\epsilon$ and $L \rightarrow \epsilon$ this becomes

$$
\left.S_{1}(1-\epsilon \text { or } \epsilon)\right|_{n=1}=\frac{2}{3} \log \epsilon .
$$

So, there is no contribution from $\left.\left(S_{1}(1-\epsilon)-S_{1}(\epsilon)\right)\right|_{n=1}$ to the finite part of the entanglement entropy (3.6)

For $S_{2}$ we have

$$
S_{2}(L)=-\frac{1}{1-n} \sum_{k=0}^{n-1} \log \left|\frac{W(k, n)}{2 \tau}\right|,
$$

with $\operatorname{det}(W)=2 i \operatorname{Im}\left(W_{1}^{1} W_{2}^{2}\right)$. The integrals of $W_{1}^{1}$ and $W_{2}^{2}$ in the limit $y \rightarrow 0$ are evaluated in appendix B. We get the following from (B.50) and (B.51).

$$
\begin{gathered}
W_{1}^{1}=\frac{\vartheta_{1}\left(-\frac{k}{n}\right)}{\vartheta_{1}^{\prime}(0)}, \quad W_{2}^{2}=\tau \frac{\vartheta_{1}\left(-\frac{k}{n}\right)}{\vartheta_{1}^{\prime}(0)} . \\
\operatorname{det}(W(k, n))=2 \tau\left|\frac{\vartheta_{1}\left(\frac{k}{n}\right)}{\vartheta_{1}^{\prime}(0)}\right|^{2} .
\end{gathered}
$$

The expression for $S_{2}(1)$ then becomes

$$
S_{2}(1)=-\frac{1}{1-n} \sum_{k=0}^{n-1} \log \left|\frac{\vartheta_{1}\left(\frac{k}{n}\right)}{\vartheta_{1}^{\prime}(0)}\right|^{2} .
$$

Since we are interested in the entanglement entropy, we take the $n \rightarrow 1$ limit of the above expression.

$$
\begin{aligned}
\left.S_{2}(1)\right|_{n=1} & =\left.\frac{-1}{1-n} \log \left|\prod_{k=0}^{n-1}\left(\frac{\vartheta_{1}(k / n)}{\vartheta_{1}^{\prime}(0)}\right)\right|^{2}\right|_{n=1}, \\
& =\left.\frac{\partial}{\partial n} \sum_{k=0}^{n-1} \log \left|\frac{\vartheta_{1}(k / n)}{\vartheta_{1}^{\prime}(0)}\right|^{2}\right|_{n=1}
\end{aligned}
$$


On performing a S-modular transformation, since we are interested in the high temperature expansion we get

$$
\begin{aligned}
\left.S_{2}(1)\right|_{n=1} & =\left.2 \frac{\partial}{\partial n} \sum_{k=0}^{n-1} \log \left|\frac{\vartheta_{1}\left(\frac{k}{n \tau} \mid-\frac{1}{\tau}\right)}{\vartheta_{1}^{\prime}\left(0 \mid-\frac{1}{\tau}\right)}\right|\right|_{n=1}+\left.\frac{\partial}{\partial n} \sum_{k=0}^{n-1}\left(-\frac{2 \pi i k^{2}}{n^{2} \tau}\right)\right|_{n=1}, \\
& =-\frac{\pi}{3 \beta}+\left.2 \frac{\partial}{\partial n} \sum_{k=0}^{n-1} \log \left|\frac{\vartheta_{1}\left(\frac{k}{n \tau} \mid-\frac{1}{\tau}\right)}{\vartheta_{1}^{\prime}\left(0 \mid-\frac{1}{\tau}\right)}\right|\right|_{n=1} .
\end{aligned}
$$

Let us now consider the second term above

$$
\begin{aligned}
s_{2} & =\left.2 \frac{\partial}{\partial n} \sum_{k=0}^{n-1} \log \left|\frac{\vartheta_{1}\left(\frac{k}{n \tau} \mid-\frac{1}{\tau}\right)}{\vartheta_{1}^{\prime}\left(0 \mid-\frac{1}{\tau}\right)}\right|\right|_{n=1}, \\
& =\left.2 \partial_{n} \sum_{k=0}^{n-1} \log \left|\sinh \left(\frac{\pi k}{n \beta}\right) \prod_{m=1}^{\infty} \frac{\left(1-e^{\frac{2 \pi k}{n \beta}} e^{-\frac{2 \pi m}{\beta}}\right)\left(1-e^{-\frac{2 \pi k}{n \beta}} e^{-\frac{2 \pi m}{\beta}}\right)}{\left(1-e^{-\frac{2 \pi m}{\beta}}\right)^{2}}\right|\right|_{n=1}, \\
& =2 \partial_{n} \sum_{k=0}^{n-1} \log \left|\sinh \left(\frac{\pi k}{n \beta}\right)\right|+\left.2 \partial_{n} \sum_{k=0}^{n-1} \log \left|\prod_{m=1}^{\infty} \frac{\left(1-e^{\frac{2 \pi k}{n \beta}} e^{-\frac{2 \pi m}{\beta}}\right)\left(1-e^{-\frac{2 \pi k}{n \beta}} e^{-\frac{2 \pi m}{\beta}}\right)}{\left(1-e^{-\frac{2 \pi m}{\beta}}\right)^{2}}\right|\right|_{n=1} .
\end{aligned}
$$

The second term in the last line of the above equation can be written as

$$
-\left.8 \partial_{n} \sum_{k=0}^{n-1} \sum_{m=1}^{\infty} \sum_{l=1}^{\infty} \frac{1}{l} e^{-2 \pi m l / \beta} \sinh ^{2}\left(\frac{\pi k l}{n \beta}\right)\right|_{n=1} .
$$

Performing the sums over $m$ and $k$ we get

$$
\begin{aligned}
& -\left.8 \partial_{n} \sum_{l=1}^{\infty} \frac{1}{l} \frac{e^{-2 \pi l / \beta}}{1-e^{-2 \pi l / \beta}} \frac{1}{4}\left(\frac{\sinh \left(\frac{\pi l}{\beta n}(2 n-1)\right)}{\sinh \left(\frac{\pi l}{\beta n}\right)}-2 n+1\right)\right|_{n=1}, \\
= & 4 \sum_{l=1}^{\infty} \frac{1}{l} \frac{e^{-2 \pi l / \beta}}{1-e^{-2 \pi l / \beta}}\left(1-\frac{\pi l}{\beta} \cot \left(\frac{\pi l}{\beta}\right)\right) .
\end{aligned}
$$

Therefore, the final expression for $S_{2}$ in the $n \rightarrow 1$ limit is given by

$$
\begin{aligned}
\left.S_{2}(1)\right|_{n=1} & =-\frac{\pi}{3 \beta}+\left.2 \partial_{n} \sum_{k=0}^{n-1} \log \left|\sinh \left(\frac{\pi k}{n \beta}\right)\right|\right|_{n=1}+4 \sum_{l=1}^{\infty} \frac{1}{l} \frac{e^{-2 \pi l / \beta}}{1-e^{-2 \pi l / \beta}}\left(1-\frac{\pi l}{\beta} \cot \left(\frac{\pi l}{\beta}\right)\right), \\
& =-\frac{\pi}{3 \beta}+\left(\frac{\pi}{\beta}-\frac{4 \pi}{\beta} \sum_{l=1}^{\infty} \frac{1}{e^{2 \pi l /(n \beta)}-1}\right)+4 \sum_{l=1}^{\infty} \frac{1}{l} \frac{e^{-2 \pi l / \beta}}{1-e^{-2 \pi l / \beta}}\left(1-\frac{\pi l}{\beta} \cot \left(\frac{\pi l}{\beta}\right)\right), \\
& =\frac{2 \pi}{3 \beta}-\frac{4 \pi}{\beta} \sum_{l=1}^{\infty} \frac{1}{e^{2 \pi l /(n \beta)}-1}+4 \sum_{l=1}^{\infty} \frac{1}{l} \frac{e^{-2 \pi l / \beta}}{1-e^{-2 \pi l / \beta}}\left(1-\frac{\pi l}{\beta} \cot \left(\frac{\pi l}{\beta}\right)\right) .
\end{aligned}
$$

It can be checked that for $L=\epsilon$ we have $W(k, n)=\tau=W(0,1)$. Therefore, $S_{2}(\epsilon)$ is zero from (3.9).

Thus, the finite contribution to the entanglement entropy (3.6) at $L \rightarrow 1$ is

$$
S_{\text {finite }}(L \rightarrow 1)=\frac{2 \pi}{3 \beta}-\frac{4 \pi}{\beta} \sum_{l=1}^{\infty} \frac{1}{e^{2 \pi l /(n \beta)}-1}+4 \sum_{l=1}^{\infty} \frac{1}{l} \frac{1}{e^{2 \pi l / \beta}-1}\left(1-\frac{\pi l}{\beta} \cot \left(\frac{\pi l}{\beta}\right)\right) \text {. }
$$




\section{Thermal entropy of free bosons on a torus}

We shall now calculate the thermal entropy for free bosons on a circle at finite temperature and verify whether it matches with (3.19). For a free complex boson on a circle the contribution to the partition function from the oscillator modes is given by [33]

$$
Z_{\mathrm{osc}}=\frac{1}{|\eta(\tau)|^{4}}
$$

On performing a modular transformation we get

$$
Z_{\mathrm{osc}}=\beta^{-2} e^{\frac{\pi}{3 \beta}} \prod_{n=1}^{\infty}\left|1-e^{\frac{-2 \pi n}{\beta}}\right|^{-4} .
$$

In addition to the oscillator modes we also need to take into consideration the contribution from the zero modes or the momenta. The contribution of these modes to the partition function is given by

$$
Z_{\text {kin }}=\left[\int_{-\infty}^{\infty} d p_{1} d p_{2} e^{-\beta\left(p_{1}^{2}+p_{2}^{2}\right)}\right]^{2}=\left(\frac{2 \pi}{\beta}\right)^{2} .
$$

The full partition function is then given as a product

$$
Z=Z_{\mathrm{osc}} Z_{\text {kin }}
$$

The thermal entropy can therefore be calculated as follows

$$
\begin{aligned}
S & =\beta^{2} \partial_{\beta}\left(-\beta^{-1} \log Z\right), \\
& =\frac{2 \pi}{3 \beta}-4 \sum_{n=1}^{\infty} \log \left|1-e^{\frac{-2 \pi n}{\beta}}\right|-\frac{8 \pi}{\beta} \sum_{n=1}^{\infty} \frac{n}{e^{2 \pi n / \beta}-1} .
\end{aligned}
$$

The above expression matches with (3.19). This can be seen from the following identities

$$
\begin{aligned}
\sum_{l=0}^{\infty} \frac{1}{l} \frac{1}{1-e^{-2 \pi l \beta}} & =-\sum_{n=0}^{\infty} \log \left|1-e^{-2 \pi n / \beta}\right| \\
\sum_{l=1}^{\infty} \frac{1}{e^{2 \pi l / \beta}-1} \cot \left(\frac{\pi l}{\beta}\right) & =2 \sum_{m=1}^{\infty} \frac{m}{e^{2 \pi m / \beta}-1}-\sum_{m=1}^{\infty} \frac{1}{e^{2 \pi m / \beta}-1} .
\end{aligned}
$$

This verifies the claim that the entanglement entropy reduces to the thermal entropy in the limit of the interval size equalling the size of the full spatial circle.

\section{Finite size corrections to Rényi entropies}

In this section we set up a high temperature expansion of the Rényi entropies in order to extract finite size corrections to the universal part. We first do this for bosons and then for then for the fermions. Let's define the following ${ }^{6}$

$$
\begin{aligned}
S_{n} & =S_{1}^{(n)}+S_{2}^{(n)}, \\
S_{1}^{(n)} & =-\frac{1}{1-n} \sum_{k=0}^{n-1} \frac{k}{n}\left(1-\frac{k}{n}\right) \log \left|\frac{\vartheta_{1}\left(z_{2}-z_{1}\right) \overline{\vartheta_{1}\left(z_{2}-z_{1}\right)}}{\vartheta_{1}^{\prime}(0)^{2}}\right|=\frac{n+1}{6 n} \log \left|\frac{\vartheta_{1}\left(z_{2}-z_{1}\right)}{\vartheta_{1}^{\prime}(0)}\right|^{2},
\end{aligned}
$$

\footnotetext{
${ }^{6}$ These are same definitions as in $(3.4)$ but with the extra $(n)$ superscript.
} 


$$
S_{2}^{(n)}=-\frac{1}{1-n} \sum_{k=0}^{n-1} \log \left|\frac{W(k, n)}{2 \tau}\right| .
$$

In order to evaluate the entanglement entropy we should take the $n \rightarrow 1$ limit of the above. We get

$$
\begin{aligned}
& S_{1}^{(1)}=\frac{2}{3} \log \left|\frac{\vartheta_{1}\left(z_{2}-z_{1}\right)}{\vartheta_{1}^{\prime}(0)}\right|, \\
& S_{2}^{(1)}=\left.\partial_{n}\left(\sum_{k=0}^{n-1} \log \left|\frac{W(k, n)}{2 \tau}\right|\right)\right|_{n=1} .
\end{aligned}
$$

\section{High temperature expansions of Rényi and entanglement entropies}

We are now interested in the high-temperature expansion. We shall retain just the leading order terms which will facilitate the comparison with the holographic computation. The high-temperature expansion of $W_{2}^{2}(k, n)$ is obtained in (4.7). The interval of length $L(=$ $1-2 y)$ is now defined by $z_{1}=y=\frac{1-L}{2}$ and $z_{2}=1-y=\frac{1+L}{2}$. The expressions for the Rényi and entanglement entropy are

$$
\begin{aligned}
S_{1}^{(n)} & =\frac{n+1}{3 n} \log \left|\frac{\beta}{\pi} e^{-\pi L^{2} / \beta} \sinh \left(\frac{\pi L}{\beta}\right) \prod_{m=1}^{\infty} \frac{\left(1-e^{2 \pi L / \beta} q^{m}\right)\left(1-e^{-2 \pi L / \beta} q^{m}\right)}{\left(1-q^{m}\right)^{2}}\right|, \\
& =\frac{n+1}{3 n} \log \left|\frac{\beta}{\pi} e^{-\pi L^{2} / \beta} \sinh \left(\frac{\pi L}{\beta}\right)\right|-\frac{n+1}{3 n} \sum_{m=1}^{\infty} \sum_{l=1}^{\infty} \frac{q^{m l}}{l} 4 \sinh ^{2}\left(\frac{\pi L l}{\beta}\right), \\
& =\frac{n+1}{3 n} \log \left|\frac{\beta}{\pi} e^{-\pi L^{2} / \beta} \sinh \left(\frac{\pi L}{\beta}\right)\right|-\frac{4(n+1)}{3 n} e^{-2 \pi T} \sinh ^{2}(\pi L T)+\mathcal{O}\left(e^{-4 \pi T}\right), \\
S_{1}^{(1)} & =\frac{2}{3} \log \left|\frac{\beta}{\pi} e^{-\pi L^{2} / \beta} \sinh \left(\frac{\pi L}{\beta}\right)\right|-\frac{8}{3} e^{-2 \pi T} \sinh ^{2}(\pi L T)+\mathcal{O}\left(e^{-4 \pi T}\right) .
\end{aligned}
$$

Now using the fact that $\operatorname{det}(W)=2 i \operatorname{Im}\left(W_{1}^{1} W_{2}^{2}\right)$ and $W_{2}^{2}=\tau W_{1}^{1}$ as shown in appendix B) we obtain

$$
\begin{aligned}
S_{2}^{(n)} & =-\frac{1}{1-n}\left(\sum_{k=0}^{\infty} \log \left|W_{1}^{1}(k, n)\right|+\sum_{k=0}^{\infty} \log \left|\frac{W_{2}^{2}(k, n)}{\beta}\right|\right), \\
& =-\frac{1}{1-n}\left(2 \sum_{k=0}^{\infty} \log \left|\frac{W_{2}^{2}(k, n)}{\beta}\right|\right) \\
S_{2}^{(1)} & =\partial_{n}\left(2 \sum_{k=0}^{\infty} \log \left|\frac{W_{2}^{2}(k, n)}{\beta}\right|\right) .
\end{aligned}
$$

The expression for $W_{2}^{2}$ is given by

$$
W_{2}^{2}=\int_{0}^{\tau} d \bar{z} \frac{\vartheta_{1}\left(\bar{z}-\frac{1}{2}-\frac{L}{2}+\frac{k L}{N}\right)}{\vartheta_{1}\left(\bar{z}-\frac{1}{2}+\frac{L}{2}\right)^{k / n} \vartheta_{1}\left(\bar{z}-\frac{1}{2}-\frac{L}{2}\right)^{1-k / n}} .
$$

The high temperature expansion of the above integral (in powers of $e^{-2 \pi T}$ ) is given in appendix B in equation (B.47). We can then do the sum over $k$, take the logarithm and 
take the limit $n \rightarrow 1$ to obtain the following quantities

$$
\begin{aligned}
& \sum_{k=1}^{n-1} \log \left|\frac{W_{2}^{2}(k, n)}{\beta}\right|= \frac{\pi\left(n^{2}-1\right) L^{2}}{6 \beta} \\
&+\left[2\left(n-\frac{\sinh ^{2}(\pi L / \beta)}{n \sinh ^{2}(\pi L /(n \beta))}\right)+\frac{2\left(n^{2}-1\right)}{3 n} \sinh ^{2}(\pi L / \beta)\right] e^{-2 \pi / \beta} \\
&+\mathcal{O}\left(e^{-4 \pi / \beta}\right)
\end{aligned}
$$

\subsection{Bosons}

In this sub-section we put together the high temperature expansions of $S_{1}^{(n)}$ and $S_{2}^{(n)}$ to obtain the Rényi entropies. From (4.1) and (4.6) we have the following ${ }^{7}$

$$
\begin{gathered}
S_{1}^{(n)}=\frac{n+1}{3 n} \log \left|\frac{R}{\pi T} e^{-\pi T L^{2} / R} \sinh (\pi L T)\right|-\frac{4(n+1)}{3} e^{-2 \pi T R} \sinh ^{2}(\pi L T) \\
+\mathcal{O}\left(e^{-4 \pi T R}\right), \\
S_{1}^{(1)}=\frac{2}{3} \log \left|\frac{R}{\pi T} e^{-\pi T L^{2} / R} \sinh (\pi L T)\right|-\frac{8}{3} e^{-2 \pi T R} \sinh ^{2}(\pi L T)+\mathcal{O}\left(e^{-4 \pi T R}\right) .
\end{gathered}
$$

Substituting (4.10) and (4.11) in (4.7) and (4.8) we get

$$
\begin{aligned}
S_{2}^{(n)}= & \frac{\pi(n+1) L^{2}}{3 \beta}+2\left[\frac{2}{n-1}\left(n-\frac{\sinh ^{2}(\pi L T)}{n \sinh ^{2}(\pi L T / n)}\right)+\frac{2(n+1)}{3 n} \sinh ^{2}(\pi L T)\right] e^{-2 \pi T R} \\
& +\mathcal{O}\left(e^{-4 \pi T R}\right), \\
S_{2}^{(1)}= & \frac{2 \pi T L^{2}}{3 R}+2\left[\frac{10}{3}+\frac{2}{3} \cosh (2 \pi L T)-4 \pi L T \operatorname{coth}(\pi L T)\right] e^{-2 \pi T R}+\mathcal{O}\left(e^{-4 \pi T R}\right) \\
= & \frac{2 \pi T L^{2}}{3 R}+\frac{8}{3} \sinh ^{2}(\pi L T) e^{-2 \pi T R}+8[1-\pi L T \operatorname{coth}(\pi L T)] e^{-2 \pi T R}+\mathcal{O}\left(e^{-4 \pi T R}\right) .
\end{aligned}
$$

By adding the two parts above we get the Rényi/entanglement entropy to be

$$
\begin{aligned}
& S_{n}=\frac{n+1}{3 n} \log \left|\frac{R}{\pi T} \sinh (\pi L T)\right|+\frac{4}{n-1}\left[n-\frac{\sinh ^{2}(\pi L T)}{n \sinh ^{2}(\pi L T / n)}\right] e^{-2 \pi R T}+\mathcal{O}\left(e^{-4 \pi T R}\right), \\
& S_{E}=\frac{2}{3} \log \left|\frac{R}{\pi T} \sinh (\pi L T)\right|+8[1-\pi L T \operatorname{coth}(\pi L T)] e^{-2 \pi T R}+\mathcal{O}\left(e^{-4 \pi T R}\right) .
\end{aligned}
$$

${ }^{7}$ So far we had been working in units where the length of the spatial circle $R$ is set to unity. We shall now restore $R$ by $L \rightarrow L / R$ and $\beta \rightarrow \beta / R$. 
One can keep track of terms upto any arbitrary order in $e^{-2 \pi T R}$. The expression for the entanglement entropy upto $\mathcal{O}\left(e^{-6 \pi T R}\right)$ is

$$
\begin{aligned}
& S_{E}= \frac{2}{3} \log \left|\frac{R}{\pi T} \sinh (\pi L T)\right|+8[1-\pi L T \operatorname{coth}(\pi L T)] e^{-2 \pi T R} \\
&+\left(\frac{49}{5}+\frac{14}{15} \cosh (2 \pi L T)+\frac{4}{15} \cosh (4 \pi L T)-\frac{21}{2} \pi L T \operatorname{csch}(\pi L T) \operatorname{sech}(\pi L T)\right. \\
& \quad-\frac{1}{2} \operatorname{sech}^{2}(\pi L T)-6 \pi L T \sinh (2 \pi L T) \operatorname{sech}^{2}(\pi L T) \\
&\left.+\frac{1}{2} \pi L T \tanh (\pi L T) \operatorname{sech}^{2}(\pi L T)\right) e^{-4 \pi T R}+\mathcal{O}\left(e^{-6 \pi T R}\right)
\end{aligned}
$$

The low temperature expansion of the Rényi and entanglement entropies can be obtained directly starting from the low temperature expansions of the twist correlators and the integrals of the cut differentials or by the following replacements in (4.16) and (4.18)

$$
R \rightarrow \frac{i}{T}, \quad T \rightarrow-\frac{i}{R}
$$

These replacements implement the modular transformation relating the low and high temperature expansions. The derivative of the Rényi entropy in the limit $n \rightarrow 1$ is universal in a zero temperature CFT and is proportional to the central charge [34]. However we see that for a finite temperature finite size system of free bosons the derivative of Rényi entropy is given by

$$
\begin{aligned}
\left.S_{n}^{\prime}\right|_{n \rightarrow 1}= & -\frac{1}{3} \log \left|\frac{R}{\pi T} \sinh (\pi L T)\right| \\
& -4 e^{-2 \pi T R}\left(1+2 \pi^{2} L^{2} T^{2}+\pi L T\left(3 \pi L T \operatorname{csch}^{2}(\pi L T)-4 \operatorname{coth}(\pi L T)\right)\right) \\
& +\mathcal{O}\left(e^{-4 \pi T R}\right) .
\end{aligned}
$$

Therefore there are finite size corrections to this universal property of the Rényi entropy. ${ }^{8}$

\subsection{Fermions}

We would like to consider the entanglement entropy of fermions on a circle and then check whether the finite size corrections can be reproduced by one-loop calculations in the bulk. The entanglement and Rényi entropies were calculated in $[14,15]$. We quote the answers here. The high temperature expansion of the Rényi entropy is given by

$$
\begin{aligned}
S^{(n)} & =S_{1}^{(n)}+S_{2}^{(n)} \\
S_{1}^{(n)} & =\frac{n+1}{6 n} \log \left|\frac{\beta}{\pi} e^{-\pi L^{2} / \beta} \sinh \left(\frac{\pi L}{\beta}\right) \prod_{m=1}^{\infty} \frac{\left(1-e^{2 \pi L / \beta} q^{m}\right)\left(1-e^{-2 \pi L / \beta} q^{m}\right)}{\left(1-q^{m}\right)^{2}}\right| \\
S_{2}^{(n)} & =\frac{n+1}{6 n} \frac{\pi L^{2}}{\beta}-\frac{2}{1-n} \sum_{j=1}^{\infty} \frac{(-1)^{\nu j}}{j} \frac{1}{\sinh (\pi j / \beta)}\left(\frac{\sinh (\pi j L)}{\sinh (\pi j L /(n \beta))}-n\right)
\end{aligned}
$$

\footnotetext{
${ }^{8}$ We thank Aninda Sinha for raising this point.
} 
where $\nu(=1,2,3,4)$ denotes the spin structure. We shall work with $\nu=2$ or (R,NS) fermions, which has the boundary conditions

$$
\psi(z+1)=\psi(z), \quad \psi(z+\tau)=-\psi(z) .
$$

To a first few leading orders the Rényi entropy is

$$
\begin{aligned}
S^{(n)}= & \frac{n+1}{6 n} \log \left|\frac{R}{\pi T} \sinh \left(\frac{\pi L}{\beta}\right)\right|-\frac{2(n+1)}{3 n} e^{-2 \pi R T} \sinh ^{2}(\pi L T) \\
& -\frac{4}{1-n}\left(\frac{\sinh (\pi L)}{\sinh (\pi L T / n)}-n\right) e^{-\pi R T}-\frac{2}{1-n}\left(\frac{\sinh (2 \pi L)}{\sinh (2 \pi L T / n)}-n\right) e^{-2 \pi R T} \\
& +\mathcal{O}\left(e^{-3 \pi R T}\right) .
\end{aligned}
$$

The EE given as the sum $S_{E}=S_{1}+S_{2}$.

$$
S_{1}=\frac{1}{3} \log \left|\frac{\beta}{\pi} e^{-\pi L^{2} / \beta} \sinh \left(\frac{\pi L}{\beta}\right) \prod_{m=1}^{\infty} \frac{\left(1-e^{2 \pi L / \beta} q^{m}\right)\left(1-e^{-2 \pi L / \beta} q^{m}\right)}{\left(1-q^{m}\right)^{2}}\right|
$$

here, $q=e^{2 \pi / \beta}$. The leading order behaviour at high-temperatures is then (the circumference of the circle $R$ is restored)

$$
S_{1}=\frac{1}{3} \log \left|\frac{R}{\pi T} \sinh \left(\frac{\pi L}{\beta}\right)\right|-\frac{\pi T L^{2}}{3 R}-\frac{4}{3} e^{-2 \pi R T} \sinh ^{2}(\pi L T)+\mathcal{O}\left(e^{-3 \pi R T}\right) .
$$

The expression for $S_{2}$ is as follows

$$
S_{2}=\frac{\pi T L^{2}}{3 R}-2 \sum_{i=1}^{\infty} \frac{1}{l}\left[1-\frac{\pi L l}{\beta} \operatorname{coth}\left(\frac{\pi L l}{\beta}\right)\right] \frac{1}{\sinh \left(\frac{\pi l}{\beta}\right)} .
$$

The leading order behaviour of $S_{2}$ is

$$
\begin{array}{r}
S_{2}=\frac{\pi T L^{2}}{3 R}-4[1-\pi L T \operatorname{coth}(\pi L T)] e^{-\pi R T}-2[1-2 \pi L T \operatorname{coth}(2 \pi L T)] e^{-2 \pi R T} \\
+\mathcal{O}\left(e^{-3 \pi R T}\right) .
\end{array}
$$

Thus, the entanglement entropy at the first few leading orders at high-temperatures is

$$
\begin{aligned}
S_{E}= & \frac{1}{3} \log \left|\frac{R}{\pi T} \sinh \left(\frac{\pi L}{\beta}\right)\right|-4[1-\pi L T \operatorname{coth}(\pi L T)] e^{-\pi R T} \\
& -2[1-2 \pi L T \operatorname{coth}(2 \pi L T)] e^{-2 \pi R T}-\frac{4}{3} e^{-2 \pi R T} \sinh ^{2}(\pi L T)+\mathcal{O}\left(e^{-3 \pi R T}\right) .
\end{aligned}
$$

Note that just as in the case of bosons, the low temperature expansions for the fermions can be obtained by the replacements given in equation (4.19) in the expressions for the Rényi entropies and entanglement entropies (4.24) and (4.29).

In the next section we shall show that the leading order one-loop corrections calculated from the bulk exactly reproduce these finite size corrections in (4.16), (4.18), (4.24) and (4.29). 


\section{$5 \quad$ Finite size corrections from holography}

As mentioned before, in order to evaluate the Rényi entropies we use the replica trick which involves finding the partition function $Z_{n}$ on the $n$-sheeted cover or a Riemann surface $\Sigma=\mathbb{C} / \Gamma$ with genus $g$. In the dual gravitational theory, we need to evaluate the partition function on a quotient $A d S_{3} / \Gamma$ which has $\Sigma$ as its conformal boundary [23, 24, 26]. It was shown in [26] that in order to obtain the one-loop contribution from the bulk we need to find the Schottky uniformization of the corresponding branched cover $(\Sigma=\mathbb{C} / \Gamma)$ and then calculate the one-loop partition function in the bulk quotient $A d S_{3} / \Gamma$. In this section we shall first review the setup for the computation developed in [26] and then utilize it to calculate the one-loop corrections to the entanglement entropy for bosons and fermions from the bulk.

\section{Holographic computation of Rényi entropies for one interval on a torus}

Since we are interested in a system at finite temperature we require to Euclideanize the temporal direction with a period having the inverse temperature. The spatial direction is also periodic. This gives a torus with two different periodicities. If $z$ is the coordinate on the torus we then have the following doubly-periodic identifications

$$
z \rightarrow z+R \mathbb{Z}+\frac{i \mathbb{Z}}{T}
$$

Note that this identification is invariant under the exchange

$$
R \rightarrow \frac{i}{T}, \quad T \rightarrow-\frac{i}{R}
$$

This property turns out to be useful while obtaining high-temperature expansions from low-temperature ones (and vice-versa) of modular invariant quantities.

Our first task is to find the discrete group $\Gamma$ by which we can form $A d S_{3} / \Gamma$ which has $\Sigma=\mathbb{C} / \Gamma$ as its conformal boundary. Every compact Riemann surface $(\Sigma)$ can be obtained as $\mathbb{C} / \Gamma$ with $\Gamma$ being the Schottky group. The Schottky group is a discrete sub-group of $\operatorname{PSL}(2, \mathbb{C})$. For of a genus $g$ Riemann surface, it is generated by $g$ loxodromic generators, $L_{i}$ where $i=1,2, \cdots, g$. It was shown in [26] that for the case of the torus the differential equation for Schottky uniformization is

$$
\psi^{\prime \prime}(z)+\frac{1}{2} \sum_{i=1}^{2}\left(\Delta \wp\left(z-z_{i}\right)+\gamma(-1)^{i+1} \zeta\left(z-z_{i}\right)+\delta\right) \psi(z)=0 .
$$

where $\gamma$ is the accessory parameter, $\Delta=\frac{1}{2}\left(1-\frac{1}{n^{2}}\right), \wp$ is the Weierstrass elliptic function and $\zeta$ is the Weierstrass zeta function. The generators of the Schottky group can be obtained by solving the monodromy problem of the solutions $\psi$ of the above equation. This will be outlined below.

The derivative of the entanglement entropy is given in terms of accessory parameter as

$$
\frac{\partial S_{E}}{\partial z_{i}}=-\lim _{n \rightarrow 1} \frac{c n}{6(n-1)} \gamma_{i}
$$




\section{Classical result}

In order to obtain the classical contribution we need to find the accessory parameter in the torus differential equation (5.2) and then use (5.3). Expanding in $\epsilon(\equiv n-1)$

$$
\psi(z)=\psi_{(0)}+\epsilon \psi_{(1)}, \quad \Delta=\epsilon, \quad \gamma=\epsilon \gamma_{(1)}, \quad \delta=-(\pi T)^{2}+\epsilon \delta_{(1)} .
$$

The zeroth and first order solutions are obtained as

$$
\begin{aligned}
& \psi_{(0)}(z)=A e^{z \pi T}+B e^{-z \pi T} \\
& \psi_{(1)}(z)=\frac{e^{-z \pi T}}{2 \pi T} \int_{0}^{z} e^{x \pi T} m(x) \psi_{(0)}(x) d x-\frac{e^{z \pi T}}{2 \pi T} \int_{0}^{z} e^{-x \pi T} m(x) \psi_{(0)}(x) d x,
\end{aligned}
$$

where

$$
m(z)=\frac{1}{2} \sum_{i=1}^{2}\left(\Delta \wp\left(z-z_{i}\right)+\gamma_{(1)}(-1)^{i+1} \zeta\left(z-z_{i}\right)+\delta_{(1)}\right) .
$$

By demanding that the first order solution has trivial monodromy around the time circle, one can find the value of the accessory parameter to be

$$
\gamma_{(1)}=2 \pi T \operatorname{coth} \pi T\left(z_{2}-z_{1}\right)
$$

Using (5.3) we get the entanglement entropy

$$
S_{E}=\frac{c}{6} \log \left|\sinh ^{2} \pi T\left(z_{2}-z_{1}\right)\right|+\text { const. }
$$

\section{One-loop corrections}

One-loop determinants on quotients of $A d S_{3}$ have been obtained in [31]. The steps followed in order to obtain the one-loop corrections to the entanglement entropy is as follows.

1. The Schottky group $\Gamma$ corresponding to $n$-sheeted cover is found. This is done by solving the monodromy problem and then finding the loxodromic generators $L_{i}$ of the group.

2. The set of representatives of the primitive conjugacy classes $(\gamma \in \mathcal{P})$ of $\Gamma$ are then found by forming non-repeated words from the $L_{i}$ and their inverses upto conjugation in $\Gamma$.

3. Largest eigenvalues of the words $\left(q_{\gamma}\right)$ in each primitive conjugacy class $\gamma$ are then computed and substituted into the expressions for the one-loop determinants. We then sum over all primitive conjugacy classes

$$
\left.\log Z[n]\right|_{\text {one-loop }}=\sum_{\gamma \in \mathcal{P}} \log Z\left(q_{\gamma}\right)
$$

where $Z(q)$ is the one loop determinant of the bulk fields in thermal $A d S_{3}$ with modular parameter $q$. 
4. The one-loop correction to the Rényi entropy can found using

$$
\left.S_{n}\right|_{\text {one-loop }}=\frac{1}{1-n}\left(\left.\log Z[n]\right|_{\text {one-loop }}-\left.n \log Z[1]\right|_{\text {one-loop }}\right) .
$$

The correction to the entanglement entropy is then obtained by taking the $n \rightarrow 1$ of the above answer.

We shall choose the interval as $[-y, y]$ i.e. the length of the interval is $L=2 y$. We shall calculate the one-loop correction in the high-temperature limit. We define a new coordinate $u$ as

$$
u \equiv e^{-2 \pi T z}
$$

Defining $u_{y}=e^{2 \pi T y}, u_{R}=e^{2 \pi T R}$ and $f(u)=u+u^{-1}$ we can write the Weierstrass elliptic function as

$$
\wp(z \pm y)=\sum_{m=-\infty}^{\infty} \frac{4 \pi^{2} T^{2}}{f\left(u u_{y}^{ \pm 1} u_{R}^{m}\right)}-\sum_{m \neq 0} \frac{4 \pi^{2} T^{2}}{f\left(u_{R}^{m}\right)}+\frac{\pi^{2} T^{2}}{3}
$$

and a similar expression for $\zeta(z \pm y)$.

The torus differential equation is then solved for one spatial period $-R / 2 \geq \operatorname{Re} z \geq$ $R / 2$. The ansatz for the solutions is taken as

$$
\psi^{ \pm}=\frac{1}{\sqrt{u}}\left(u-u_{y}\right)^{\Delta_{ \pm}}\left(u-\frac{1}{u_{y}}\right)^{\Delta_{\mp}} \sum_{m=-\infty}^{\infty} \psi^{ \pm(m)}\left(u_{y}, u_{R}\right) u^{m}
$$

where $\Delta_{ \pm}=\frac{1}{2}\left(1 \pm \frac{1}{n}\right)$ and the solutions are normalized as $\psi^{ \pm(0)}=1$. The coefficients $\psi^{ \pm(m)}$ are then expanded in a power series in $u_{R}$ in the following form

$$
\psi^{ \pm(m)}\left(u_{y}, u_{R}\right)=\sum_{k=|m|}^{\infty} \psi^{ \pm(m, k)}\left(u_{y}\right) u_{R}^{k}
$$

The differential equation (5.2) is then expanded in $u$ and $u_{R}$. The coefficients $\psi^{ \pm(m, k)}$ can then be determined. Also one can obtain $\gamma$ and $\delta$ upto any order in $u_{R}$

$$
\begin{aligned}
& \gamma=\pi T\left(1+u_{y}^{2}\right)\left[\frac{1-n^{2}}{n^{2}\left(u_{y}^{2}-1\right)}+\frac{\left(n^{2}-1\right)^{2}\left(u_{y}^{2}-1\right)^{3}}{6 n^{4} u_{y}^{4}} u_{R}^{2}+\mathcal{O}\left(u_{R}^{4}\right)\right], \\
& \delta=\frac{\pi^{2} T^{2}}{n^{2}\left(u_{y}^{2}-1\right)}\left\{\frac{1}{6}\left[\left(n^{2}-1\right)\left(u_{y}^{2}+1\right) \log \left(u_{y}\right)-\left(7 n^{2}-1\right)\left(u_{y}^{2}-1\right)\right]+\mathcal{O}\left(u_{R}^{2}\right)\right\} .
\end{aligned}
$$

The classical Rényi entropy can then be obtained using (5.3)

$$
S_{n}=\frac{c(n+1)}{12 n}\left[\log \sinh ^{2}(2 \pi T y)+\text { const. }-\frac{\left(n^{2}-1\right)}{6 n^{2}}[\cosh (8 \pi T y)-4 \cosh (4 \pi T y)] e^{-4 \pi T R}\right] .
$$


The solutions to the first few orders in $u_{R}$ is

$$
\begin{aligned}
& \psi^{+}(u)=\frac{1}{\sqrt{u}}\left(u-u_{y}\right)^{\Delta_{+}}\left(u-\frac{1}{u_{y}}\right)^{\Delta_{-}}\left[1+\frac{\left(n^{2}-1\right)\left(u_{y}^{2}-1\right)^{2} u_{R}^{2}}{24 n^{3} u^{2} u_{y}^{3}} \times\right. \\
& \left.u\left((n+1) u^{2}+n-1\right) u_{y}^{2}+u\left((n-1) u^{2}+n+1\right)-n\left(u^{4}+1\right) u_{y}+\mathcal{O}\left(u_{R}^{3}\right)\right] .
\end{aligned}
$$

The solution for $\psi^{-}$is same as the above with $u \rightarrow 1 / u$. The monodromy matrices, $L_{1}$ can then be found by

$$
\left(\begin{array}{l}
\psi^{+}\left(u / u_{R}\right) \\
\psi^{-}\left(u / u_{R}\right)
\end{array}\right)=L_{1}\left(\begin{array}{l}
\psi^{+}(u) \\
\psi^{-}(u)
\end{array}\right) .
$$

To the lowest few orders we get matrix elements to be

$$
\begin{aligned}
& \left(L_{1}\right)_{11}=\frac{n u_{y}^{1-1 / n}}{\left(1-u_{y}^{2}\right) \sqrt{u_{R}}}\left\{1-\frac{\left[(n+1) u_{y}^{2}+n-1\right]^{2}}{4 n^{2} u_{y}^{2}}+\mathcal{O}\left(u_{R}^{2}\right)\right\} \\
& \left(L_{1}\right)_{11}=\frac{n u_{y}}{\left(1-u_{y}^{2}\right) \sqrt{u_{R}}}\left\{1-\frac{\left[(n+1) u_{y}^{2}+n-1\right]\left[(n+1) u_{y}^{2}+n+1\right]}{4 n^{2} u_{y}^{2}}+\mathcal{O}\left(u_{R}^{2}\right)\right\} \\
& \left(L_{1}\right)_{21}=-\left(L_{1}\right)_{12}, \quad\left(L_{1}\right)_{22}=\left.\left(L_{1}\right)_{11}\right|_{n \rightarrow n} .
\end{aligned}
$$

The other Schottky generators $L_{i}, i=2,3, \cdots, n$ can be obtained by conjugating $L_{1}$ by $M_{2}$ around $z_{2}=y$

$$
L_{i}=M_{2}^{i-1} L_{1} M_{2}^{-(i-1)}, \quad M_{2}=\left(\begin{array}{cc}
e^{2 \pi i \Delta_{+}} & 0 \\
0 & e^{2 \pi i \Delta_{-}}
\end{array}\right) .
$$

Words of length $k$ can then be constructed as follows

$$
\begin{aligned}
& L_{k_{1}}^{\sigma_{1}} L_{k_{2}}^{\sigma_{2}} \cdots L_{k_{m}}^{\sigma_{m}}=\left[\frac{n u_{y}}{\left(1-u_{y}^{2}\right) \sqrt{u_{R}}}\right]^{m}\left[\prod_{j=1}^{m-1} \sigma_{j}\left(u_{y}^{-\sigma_{j} / n}-e^{2 \pi i\left(k_{j}-k_{j+1}\right) / n} u_{y}^{\sigma_{j+1} / n}\right)\right] \\
& \times\left(\begin{array}{c}
\sigma_{m} u_{y}^{-\sigma_{m} / n} \\
\left.-\sigma_{m} e^{-2 \pi i k_{1} / m} u_{y}^{\left(\sigma_{1}-\sigma_{m}\right) / n}-\sigma_{m} e^{2 \pi i\left(k_{m}-k_{1}\right) / n} u_{y}^{\sigma_{\sigma_{1} / n}}\right)+\mathcal{O}\left(u_{R}^{-m / 2+1}\right) .
\end{array}\right.
\end{aligned}
$$

here $j=1,2, \cdots, m$ and $\sigma_{j}= \pm 1$. The larger eigenvalue of the above word can then be found out to be

$$
q_{\gamma}^{-1 / 2}=\left[\frac{n u_{y}}{\left(1-u_{y}^{2}\right) \sqrt{u_{R}}}\right]^{m} \prod_{j=1}^{\infty} \sigma_{j}\left(u_{y}^{-\sigma_{j} / n}-e^{2 \pi i\left(k_{j}-k_{j+1}\right) / n} u_{y}^{\sigma_{j+1} / n}\right)+\mathcal{O}\left(u_{R}^{-m / 2+1}\right) .
$$

here $m+1 \rightarrow 1$. One can then substitute the above expression into the formulae for one-loop partition functions in 3-d gravity and then sum over primitive conjugacy classes (i.e. over all possible non-repeated words) (5.10). The corrections to the entanglement entropy can be obtained by using

$$
\left.S_{E}\right|_{\text {one-loop }}=\left.\lim _{n \rightarrow 1} S_{n}\right|_{\text {one-loop }}=\lim _{n \rightarrow 1}-\frac{1}{n-1}\left(\left.\log Z[n]\right|_{\text {one-loop }}-\left.\log Z[1]\right|_{\text {one-loop }}\right) .
$$




\subsection{One-loop corrections: bosons}

In the CFT we used the replica trick to evaluate the Rényi entropies. This essentially is the partition function of the CFT on the $n$-sheeted cover of the CFT on the torus. As we have argued in the introduction we expect the leading finite size corrections to the Rényi entropies of the free boson CFT should agree with the one-loop determinant of the corresponding bulk field evaluated in the handlebody geometry dual the $n$-sheeted cover of the CFT on the torus. In this section we will evaluate the one-loop determinant of bulk fields in the handlebody geometry dual to the $n$-sheet cover of the CFT and show that the leading finite size corrections evaluated in the CFT agree precisely with that from the one-loop determinants.

The free real scalar in $1+1$ dimensions is not a good primary. The well defined primary with dimension $(\Delta, \bar{\Delta})=(1,0)$ or $(0,1)$ are conserved currents constructed from the scalar. If $\phi(z, \bar{z})$ is a real scalar field, the two conserved currents are then given by

$$
J(z)=\left(\partial_{z} \phi\right)(z), \quad J(\bar{z})=\left(\partial_{\bar{z}} \phi\right)(\bar{z}) .
$$

The conservation law is then guaranteed by the equations of motion: $\partial_{\bar{z}} \partial_{z} \phi(z, \bar{z})=0$. The presence of these (anti-)holomorphic currents in the CFT is equivalent to the presence of two U(1) Chern-Simons gauge fields in the bulk. The one-loop determinant for such a spin-1 field in thermal $A d S_{3}$ can be calculated by using heat kernel methods [37, 38] and is explicitly shown in appendix $\mathrm{C}$.

$$
Z_{\text {spin-1 }}^{\text {one-loop }}=\left(\prod_{n=1}^{\infty} \frac{1}{\left(1-q^{m}\right)^{1 / 2}\left(1-\bar{q}^{m}\right)^{1 / 2}}\right)^{2} .
$$

Note that we have taken the square because the the free boson of interest is complex, therefore there are 4 Chern-Simons fields in the bulk. Using (5.24) we get

$$
q_{1}^{-1 / 2}=\frac{n u_{y}\left(u_{y}^{-1 / n}-u_{y}^{1 / n}\right)}{\left(1-u_{y}^{2}\right) \sqrt{u_{R}}}+\mathcal{O}\left(\sqrt{u_{R}}\right) .
$$

Using (5.24) and the expression for the one-loop partition function (5.27), we can obtain the one-loop partition function for the $n$-sheeted cover from (5.10). We need to sum only over all single-letter words to obtain corrections upto the leading order

$$
\left.\log Z_{n}\right|_{\text {one-loop }}=\sum_{\gamma \in \mathcal{P}} \operatorname{Re}\left[2 q_{\gamma}+\mathcal{O}\left(q_{\gamma}^{2}\right)\right]=\frac{4 \sinh ^{2}(2 \pi T y)}{n \sinh ^{2}(2 \pi T y / n)} e^{-2 \pi T R}+\mathcal{O}\left(e^{-4 \pi T R}\right) .
$$

The one-loop correction to the Rényi entropy is

$$
S_{n}=\frac{4}{n-1}\left[n-\frac{\sinh ^{2}(\pi T L)}{n \sinh ^{2}(\pi T L / n)}\right] e^{-2 \pi T R}+\mathcal{O}\left(e^{-4 \pi T R}\right) .
$$

Finally, using (5.25) we can calculate the one-loop correction to the entanglement entropy

$$
\left.S_{E}\right|_{\text {one-loop }}=8(1-\pi T L \operatorname{coth}(\pi T L)) e^{-2 \pi T R}+\mathcal{O}\left(e^{-4 \pi T R}\right) .
$$

(where $L=2 y$ ). This matches precisely with the leading order correction to the universal part of the entanglement entropy obtained in (4.18). 


\subsection{One loop corrections: fermions}

In this sub-section we repeat the analysis of the comparison of one-loop determinants in the handlebody geometry to the finite size corrections evaluated in the CFT for the case of free fermions. We will see that the leading and the next-to leading finite size corrections agree evaluated in the CFT agree with the bulk one-loop determinant. Demonstrating this involves summing over two letter words to evaluate the bulk determinant. Since $(i \not \partial)^{2}=-\nabla_{(1 / 2)}^{2}$, the one-loop partition function for the spin- $\frac{1}{2}$ fermion is

$$
Z_{\text {spin- } 1 / 2}^{\text {onelop }}=\operatorname{det}(i \not \partial)=\sqrt{\operatorname{det}\left(-\nabla_{(1 / 2)}^{2}\right)} .
$$

Determinants of laplacians for fermions in the BTZ black hole were calculated using quasinormal modes and also by integrating the heat-kernel in [39]. The one-loop partition function of the bulk field dual to operators of conformal dimension $(\Delta, \bar{\Delta})=\left(\frac{1}{2}, 0\right)$ and $\left(0, \frac{1}{2}\right)$ reads

$$
Z_{\text {spin- } 1 / 2}^{\text {one-loop }}=\prod_{n=0}^{\infty}\left|1-q^{n+1 / 2}\right|^{2(n+1)} .
$$

The high temperature expansion of the one-loop determinant on the $n$-sheeted cover (5.10) reads as

$$
\left.\log Z_{n}\right|_{\text {one-loop }}=\sum_{\gamma \in \mathcal{P}} \operatorname{Re}\left[-2 q_{\gamma}^{1 / 2}-q_{\gamma}+\mathcal{O}\left(q_{\gamma}^{2}\right)\right] .
$$

We have kept the first two leading orders in the high-temperature expansion. At $\mathcal{O}\left(e^{-\pi R T}\right)$, the contribution is just from the sum over single letter words. However at $\mathcal{O}\left(e^{-2 \pi R T}\right)$, there is one contribution from the sum over two-letter words from $-2 q_{\gamma}^{1 / 2}$ and another from the sum over the squares of the single letter words from $-q_{\gamma}$.

While summing over 2-letter words $\left(L_{k_{1}}^{\sigma_{1}} L_{k_{2}}^{\sigma_{2}}\right)$ we need to keep the following constraints in mind

1. Since the sum is over primitive conjugacy classes (or non-repeated words), we need to impose $k_{1} \neq k_{2}$. Another way to say this is that, we would be summing over squares of single-letter words if $k_{1}=k_{2}$ which is already taken care of by terms in one-loop partition function which are higher order in $q_{\gamma}$.

2. We need to ensure that each $\left(k_{1}, k_{2}\right)$ pair in the sum is counted only once. This is because $L_{k_{1}}^{\sigma_{1}} L_{k_{2}}^{\sigma_{2}}$ and $L_{k_{2}}^{\sigma_{2}} L_{k_{1}}^{\sigma_{1}}$ are simply related by conjugation and hence belong to the same conjugacy class.

3. Words and their inverses cannot be related by conjugation and need to summed over separately.

The high-temperature expansion can then be written as (the prime on the $k_{2}$ sum indicates the constraints above)

$$
\begin{gathered}
\left.\log Z_{n}\right|_{\text {one-loop }}=-2 \sum_{k=0}^{n-1} \operatorname{Re}\left[q_{k}^{1 / 2}\right]-2 \sum_{k_{1}=0}^{n-1} \sum_{k_{2}=0}^{n-1} \sum_{\sigma_{1}, \sigma_{2}} \operatorname{Re}\left[\left(q_{k_{1}, k_{2}}^{\sigma_{1}, \sigma_{2}}\right)^{1 / 2}\right] \\
-\sum_{k=0}^{n-1} \operatorname{Re}\left[q_{k}\right]+\mathcal{O}\left(e^{-3 \pi R T}\right) .
\end{gathered}
$$


Let us now list eigenvalues of all possible 2-letter words. The 2-letter words are of the form $L_{k_{1}}^{\sigma_{1}} L_{k_{2}}^{\sigma_{2}}$ with $\sigma_{1,2}= \pm 1$ and $k_{1,2}=0,1, \cdots, n-1$.

$$
\begin{gathered}
\left(q_{k_{1}, k_{2}}^{ \pm \pm}\right)^{-1 / 2}=\frac{n^{2}}{\left(u_{y}^{-1}-u_{y}\right)^{2} u_{R}}\left(u_{y}^{-1 / n}-e^{2 \pi i\left(k_{1}-k_{2}\right) / n} u_{y}^{1 / n}\right)\left(u_{y}^{-1 / n}-e^{-2 \pi i\left(k_{1}-k_{2}\right) / n} u_{y}^{1 / n}\right) \\
+\mathcal{O}(1), \\
\left(q_{k_{1}, k_{2}}^{ \pm \mp}\right)^{-1 / 2}=-\frac{n^{2}}{\left(u_{y}^{-1}-u_{y}\right)^{2} u_{R}}\left(1-e^{2 \pi i\left(k_{1}-k_{2}\right) / n}\right)\left(1-e^{-2 \pi i\left(k_{1}-k_{2}\right) / n}\right)+\mathcal{O}(1) .
\end{gathered}
$$

The sums over the two-letter words in (5.35) are done in appendix D. We then have the following result for the one-loop partition function.

$$
\begin{aligned}
\left.\log Z_{n}\right|_{\text {one-loop }}= & -4 \frac{\sinh (2 \pi T y)}{\sinh (2 \pi T y / n)} e^{-\pi T R}-2\left[\frac{\sinh (4 \pi T y)}{\sinh (4 \pi T y / n)}-\frac{\sinh ^{2}(2 \pi T y)}{n \sinh ^{2}(2 \pi T y / n)}\right] e^{-2 \pi T R} \\
& +\frac{2\left(n^{2}-1\right)}{3 n} \sinh ^{2}(2 \pi T y) e^{-2 \pi T R}-\frac{2 \sinh ^{2}(2 \pi T y)}{n \sinh ^{2}(2 \pi T y / n)} e^{-2 \pi T R}+\mathcal{O}\left(e^{-3 \pi T R}\right) .
\end{aligned}
$$

The first term on the r.h.s. is the single letter word contribution from the $q_{k}^{1 / 2}$ sum. The second and third term arises from the two-letter word sums $\left(q_{k_{1}, k_{2}}^{ \pm \pm}\right)^{1 / 2}$ and $\left(q_{k_{1}, k_{2}}^{ \pm \mp}\right)^{1 / 2}$ respectively. The fourth term is the single letter word sum over $q_{k}$. Upon simplification, we get

$$
\begin{aligned}
\left.\log Z_{n}\right|_{\text {one-loop }}= & -4 \frac{\sinh (2 \pi T y)}{\sinh (2 \pi T y / n)} e^{-\pi T R}-2 \frac{\sinh (4 \pi T y)}{\sinh (4 \pi T y / n)} e^{-2 \pi T R} \\
& +\frac{2\left(n^{2}-1\right)}{3 n} \sinh ^{2}(2 \pi T y) e^{-2 \pi T R}+\mathcal{O}\left(e^{-3 \pi R T}\right) .
\end{aligned}
$$

The one-loop correction to the Rényi entropy can then be calculated to be

$$
\begin{aligned}
\left.S_{n}\right|_{\text {one-loop }}= & -\frac{1}{n-1}\left[4\left(n-\frac{\sinh (2 \pi T y)}{\sinh (2 \pi T y / n)}\right) e^{-\pi R T}+2\left(n-\frac{\sinh (4 \pi T y)}{\sinh (4 \pi T y / n)}\right) e^{-2 \pi R T}\right] \\
& -\frac{2(n+1)}{3 n} \sinh ^{2}(2 \pi T y) e^{-2 \pi R T}+\mathcal{O}\left(e^{-3 \pi R T}\right) .
\end{aligned}
$$

Taking the $n \rightarrow 1$ limit, we can calculate the one-loop correction to the entanglement entropy

$$
\begin{aligned}
\left.S_{E}\right|_{\text {one-loop }}= & -4[1-\pi T L \operatorname{coth}(\pi T L)] e^{-\pi R T}-2[1-2 \pi L T \operatorname{coth}(2 \pi T L)] e^{-2 \pi R T} \\
& -\frac{4}{3} e^{-2 \pi R T} \sinh ^{2}(\pi T L)+\mathcal{O}\left(e^{-3 \pi R T}\right) .
\end{aligned}
$$

Again, these one-loop contributions to the Rényi and entanglement entropies agree precisely with the leading order correction to the universal part for the fermion case (4.24) and (4.29). 


\subsection{Discussion}

At the first sight it might seem surprising that the one-loop corrections to the Rényi entropies from holography, which usually describes strongly coupled physics, exactly reproduces the leading order finite size corrections of free bosonic and fermionic CFT's. But as discussed in the introduction, one loop corrections of bulk fields in the handlebody geometries are sensitive to only the quantum numbers of the spectrum in the bulk and are insensitive to interactions. The one loop corrections in the bulk can be obtained just by summing the contributions over the spectra in the bulk. The one loop determinant of the Chern-Simons field in the handlebody geometries can be obtained by substituting (5.27) into (5.10) as we have done in subsection 5.1. Similarly the corresponding equation for the Dirac fermion are given by substituting (5.34) into (5.10). Note that these one-loop determinants organize into representations of Virasoro characters. The leading finite size expansions of the one-loop contributions given in (5.30) and (5.39) for the Chern-Simons field and for the Dirac fermions respectively. The contribution to finite size corrections from the classical part of the action given in (5.18) is clearly sub-leading to the leading terms from the one-loop terms. The one loop contribution to the partition function is independent of the central charge or the 3-dimensional Newton's constant and just depends on the quantum numbers of the spectrum. Therefore from the usual rules of AdS/CFT, as summarized in the equality of the partition functions in equation (1.6) we expect the leading terms in (5.30) for the Chern-Simons field and (5.39) for the Dirac field to agree with the leading finite size corrections evaluated in the CFT given in (4.16) and (4.24). We have shown in this paper by explicit computation of these correction both from the bulk and in the CFT that this expectation is borne out. This agreement is certainly a non-trivial test of the methods proposed by [26] to evaluate finite size corrections in the bulk.

Note that the $1 / c$ corrections to the partition functions in the bulk which occur at higher loops are sensitive to the OPE's of the dual CFT and certainly finite size corrections from these would not in general agree with the free boson or the free fermion CFT [31]. Another point worth mentioning is that we expect the leading classical contribution to the Rényi entropies from the bulk which is proportional to $c$ for which few terms were evaluated in (5.18) to be universal for large $c$ interacting CFT's. The reason is that this term just depends on just on the classical action of the handlebody geometry or in other words the metric of the background. It will be interesting to reproduce the classical action including the finite size corrections from this term by studying large $c$ CFT's using methods similar to [41]. This work obtained the universal features of the Rényi entropies of two intervals in large $c$ CFT's and showed that it reduces to the method proposed by [23] in the bulk.

\section{Conclusions}

In this paper we have calculated the Rényi entropies for the free boson on a circle at finite temperature using two point correlation functions of twist field operators. The answer (2.89) is expressed in terms of Jacobi elliptic theta functions and the Riemann-Siegel function which can be written as the $A_{n-1}$ lattice sum. We have also obtained the entanglement and Rényi entropies in the decompactification regime and have numerically plotted 
them. The behaviour of the entanglement entropy was then analysed in the limit of large intervals (equalling the size of the system) and it was shown to match with the thermal entropy as expected. We have then set up a systematic high temperature expansion for the Rényi entropies and have extracted the leading order finite-size corrections to the universal part of the Rényi and entanglement entropies in the high temperature expansion. As discussed in the introduction and also in section 5.3 these leading corrections from the free boson CFT are expected to agree with the leading finite size contributions to the Rényi entropies from the one-loop determinant of the Chern-Simons field in the handlebody geometries dual to the CFT on $n$-sheeted Riemann surfaces. These corrections were evaluated from the bulk using methods developed recently in [26] and were shown to agree precisely with that from the free boson CFT. We have also investigated the case of fermions in which the Rényi and entanglement entropies were calculated previously in $[14,15]$. We have shown that the leading and the next to leading order finite size corrections to the universal piece agree with that obtained from holography. This involved an agreement up to two letter words composed out of the Schottky generators.

The above analysis of free bosons along with the one for free fermions in [15] gives results for the entanglement entropy on the torus for two of the most well-studied and simplest conformal field theories in $1+1$ dimensions. These results will be useful to find entanglement and Rényi entropies of superconformal field theories in $1+1$ dimensions like the D1/D5 system which is one of the well studied examples of $A d S_{3} / C F T_{2}$. In fact it will be interesting to figure out how the dependence on the size of the target space torus $\mathcal{R}$ can be seen in the bulk.

These results also serve as a test for the holographic methods using Schottky uniformization developed in $[23,26]$ for the case of finite temperature CFT's with a compact spatial direction. The calculation of Rényi entropies require the evaluation of partition functions of CFT's on higher genus surfaces. Therefore, as emphasized in the introduction our results also test the proposals of $[30,31]$ for the evaluation of one-loop contributions to partition functions of bulk fields in handlebody geometries.

The result for Rényi entropies for the free boson CFT can also be applied to a wide class of one-dimensional statistical models at criticality which are described by $c=1$ or bosonic CFT's. Examples include one-dimensional Bose gases, Heisenberg spin chains, the 4-state Potts model and $\mathrm{Z}_{4}$ parafermions. In this regard the dependence on the size of the spacetime torus $\mathcal{R}$ will play an important role. These results might also serve as approximations in more realistic systems of bosons in 1-dimension at the critical point like trapped cold atoms, superfluids in nanopores and superconducting wires [40]. These systems are of both theoretical and experimental interest in recent times.

We end with some future directions and some open questions. One can hope to generalize the results of this work for the case of two or more disjoint intervals and then extract meaningful quantities like the mutual information. In fact correlators involving arbitrary insertions of bosonic twist fields on the torus have been evaluated in [18]. Another useful direction is to obtain exact results for the entanglement entropy for the free boson in presence of chemical potentials along the similar lines of what is done for the case of fermions [15]. Holographic studies of the situation with chemical potentials and generalization to CFT's in higher dimensions is also an interesting direction to explore [42, 43]. 


\section{Acknowledgments}

We are grateful to Rajesh Gopakumar for stimulating discussions and useful suggestions. We also thank Sachin Jain, Shaon Sahoo, Ashoke Sen and Aninda Sinha for useful discussions. We thank Sandeep Chatterjee and Abhishek Iyer for help with the numerics. J.R.D thanks the string theory group at Harish-Chandra Research Institute, Allahabad for hospitality during which part of the work was done, an opportuntity to present this work and for the stimulating discussions during the presentation. The work of J.R.D is partially supported by the Ramanujan fellowship DST-SR/S2/RJN-59/2009.

\section{A Jacobi theta functions}

In this appendix we shall list some identites involving Jacobi theta functions which are used throughout the paper. The basic theta functions are defined by the series

$$
\vartheta(z \mid \tau)=\sum_{n=-\infty}^{\infty} \exp \left(\pi i n^{2} \tau+2 \pi i n z\right)
$$

The product representations for theta functions with different characteristics or spin structures are:

$$
\begin{aligned}
& \vartheta_{1}(z \mid \tau)=2 e^{\pi i \tau / 4} \sin (\pi z) \prod_{m=1}^{\infty}\left(1-q^{m}\right)\left(1-y q^{m}\right)\left(1-y^{-1} q^{m}\right), \\
& \vartheta_{2}(z \mid \tau)=2 e^{\pi i \tau / 4} \cos (\pi z) \prod_{m=1}^{\infty}\left(1-q^{m}\right)\left(1+y q^{m}\right)\left(1+y^{-1} q^{m}\right), \\
& \vartheta_{3}(z \mid \tau)=\prod_{m=1}^{\infty}\left(1-q^{m}\right)\left(1+y q^{m}\right)\left(1+y^{-1} q^{m}\right), \\
& \vartheta_{4}(z \mid \tau)=\prod_{m=1}^{\infty}\left(1-q^{m}\right)\left(1-y q^{m}\right)\left(1-y^{-1} q^{m}\right),
\end{aligned}
$$

where $q=e^{2 \pi i \tau}$ and $y=e^{2 \pi i z}$ is used. ${ }^{9}$

The functions have the following (quasi-)periodicity properties

$$
\begin{aligned}
& \vartheta_{\nu}(z+1 \mid \tau)=\vartheta_{\nu}(z \mid \tau), \\
& \vartheta_{\nu}(z+\tau \mid \tau)=e^{-\pi i \tau-2 \pi i z} \vartheta_{\nu}(z \mid \tau) \quad \text { for } \nu=1,2,3,4 .
\end{aligned}
$$

\footnotetext{
${ }^{9}$ Note that Mathematica uses the older convention for the nome, $q=e^{\pi i \tau}$ in EllipticTheta.
} 
The S-modular transformation identities are

$$
\begin{aligned}
& \vartheta_{1}(z \mid \tau)=-i(-i \tau)^{-1 / 2} e^{-\pi i z^{2} / \tau} \vartheta_{1}\left(\frac{z}{\tau} \mid-\frac{1}{\tau}\right), \\
& \vartheta_{2}(z \mid \tau)=(-i \tau)^{-1 / 2} e^{-\pi i z^{2} / \tau} \vartheta_{4}\left(\frac{z}{\tau} \mid-\frac{1}{\tau}\right), \\
& \vartheta_{3}(z \mid \tau)=(-i \tau)^{-1 / 2} e^{-\pi i z^{2} / \tau} \vartheta_{3}\left(\frac{z}{\tau} \mid-\frac{1}{\tau}\right), \\
& \vartheta_{4}(z \mid \tau)=(-i \tau)^{-1 / 2} e^{-\pi i z^{2} / \tau} \vartheta_{2}\left(\frac{z}{\tau} \mid-\frac{1}{\tau}\right) .
\end{aligned}
$$

These functions also have the following half-periodicity properties

$$
\begin{aligned}
& \vartheta_{2}(z \mid \tau)=-\vartheta_{1}(z-1 / 2 \mid \tau) \\
& \vartheta_{3}(z \mid \tau)=-y^{-1 / 2} q^{1 / 8} \vartheta_{1}(z-1 / 2-\tau / 2 \mid \tau), \\
& \vartheta_{4}(z \mid \tau)=i y^{-1 / 2} y^{1 / 8} \vartheta_{1}(z-\tau / 2 \mid \tau) .
\end{aligned}
$$

The Dedekind eta function is

$$
\eta(\tau)=e^{\pi i \tau / 12} \prod_{m=1}^{\infty}\left(1-q^{m}\right) .
$$

\section{B Evaluating $W_{1}^{1}$ and $W_{2}^{2}$}

\section{Reality properties}

In this subsection we make the following claims regarding the elements of the matrix $W$ and prove them.

$$
\left(W_{1}^{1}\right)^{*}=W_{1}^{2}, \quad\left(W_{1}^{1}\right)^{*}=W_{1}^{1}, \quad\left(W_{2}^{1}\right)^{*}=-W_{2}^{1}, \quad\left(W_{2}^{1}\right)^{*}=W_{2}^{2} .
$$

These identities imply

- $W_{1}^{1}$ and $W_{1}^{2}$ are purely real.

- $W_{2}^{1}$ and $W_{2}^{2}$ are purely imaginary.

- $\operatorname{det}(W)$ and $\operatorname{det}(\bar{W})$ are purely imaginary. $\operatorname{det}(W)=2 i \operatorname{Im}\left(W_{1}^{1} W_{2}^{2}\right)$.

We now proceed to a proof of (B.1). We list the explicit integral forms of $W_{i}^{j}$ on choosing $z_{1}=y$ and $z_{2}=1-y$ with $0 \leq y \leq \frac{1}{2}$. We also define $y_{M}=\left(1-\frac{2 k}{n}\right) y+\frac{k}{n}$.

$$
\begin{aligned}
& W_{1}^{1}(k, n)=\int_{0}^{1} d z \vartheta_{1}(z-y)^{-(1-k / n)} \vartheta_{1}(z-1+y)^{-k / n} \vartheta_{1}\left(z-y_{M}\right), \\
& W_{1}^{2}(k, n)=\int_{0}^{1} d \bar{z} \vartheta_{1}(z-\bar{y})^{-k / n} \vartheta_{1}(\bar{z}-1+y)^{-(1-k / n)} \vartheta_{1}\left(\bar{z}+y_{M}\right), \\
& W_{2}^{1}(k, n)=\int_{0}^{\tau} d z \vartheta_{1}(z-y)^{-(1-k / n)} \vartheta_{1}(z-1+y)^{-k / n} \vartheta_{1}\left(z-y_{M}\right),
\end{aligned}
$$




$$
W_{2}^{2}(k, n)=\int_{0}^{\tau} d \bar{z} \vartheta_{1}(\bar{z}-y)^{-k / n} \vartheta_{1}(\bar{z}-1+y)^{-(1-k / n)} \vartheta_{1}\left(\bar{z}+y_{M}\right)
$$

It can then be easily seen that

$$
\operatorname{det}(W(0,1))=2 i \operatorname{Im}\left(W_{1}^{1}(0,1) W_{2}^{2}(0,1)\right)=2 \tau
$$

Observe that there exists a branch cut from $[y, 1-y]$ see figure 5. Let us first calculate the value of the integral along this branch cut.

$$
I=\int_{y}^{1-y} d z \vartheta_{1}(z-y)^{p} \vartheta_{1}(z-1+y)^{q} \vartheta_{1}\left(z-y_{M}\right),
$$

where, $p$ and $q$ are some rational fractions. Taking a contour 'around' this branch cut and calling the contribution to be $J$ we have

$$
\begin{aligned}
J & =\left(\int_{y+i \epsilon}^{1-y+i \epsilon}-\int_{1-y-i \epsilon}^{y-i \epsilon}\right) d z \vartheta_{1}(z-y)^{p} \vartheta_{1}(z-1+y)^{q} \vartheta_{1}\left(z-y_{M}\right) \\
& =\text { Sum of residues }(=0)
\end{aligned}
$$

It can be seen that

$$
\int_{y+i \epsilon}^{1-y+i \epsilon} d z \vartheta_{1}(z-y)^{p} \vartheta_{1}(z-1+y)^{q} \vartheta_{1}\left(z-y_{M}\right)=e^{i q \pi} I,
$$

and

$$
\int_{1-y-i \epsilon}^{y-i \epsilon} d z \vartheta_{1}(z-y)^{p} \vartheta_{1}(z-1+y)^{q} \vartheta_{1}\left(z-y_{M}\right)=e^{i(2 p+q) \pi} I .
$$

It can then be immediately seen that for $p=-(1-k / n)$ and $q=-k / n$ that $I=0$ from (B.8). We thus have the following result

$$
\int_{y}^{1-y} d z \vartheta_{1}(z-y)^{-(1-k / n)} \vartheta_{1}(z-1+y)^{-k / n} \vartheta_{1}\left(z-y_{M}\right)=0 .
$$

Similarly, it can be also be checked that

$$
\int_{y}^{1-y} d \bar{z} \vartheta_{1}(\bar{z}-y)^{-k / n} \vartheta_{1}(\bar{z}-1+y)^{-(1-k / n)} \vartheta_{1}\left(\bar{z}+y_{M}\right)=0 .
$$

So, for $W_{1}^{a}$ which involves integration along the real line with this branch cut we conclude that they are real.

Now, consider the following integral over the spatial cycle

$$
\begin{aligned}
W_{1}^{1} & =\int_{0}^{1} d z \vartheta_{1}(z-y)^{-(1-k / n)} \vartheta_{1}(z-1+y)^{-k / n} \vartheta_{1}\left(z-y_{M}\right) \\
& =\left(\int_{0}^{y}+\int_{y}^{1-y}+\int_{1-y}^{1}\right) d z \vartheta_{1}(z-y)^{-(1-k / n)} \vartheta_{1}(z-1+y)^{-k / n} \vartheta_{1}\left(z-y_{M}\right) .
\end{aligned}
$$




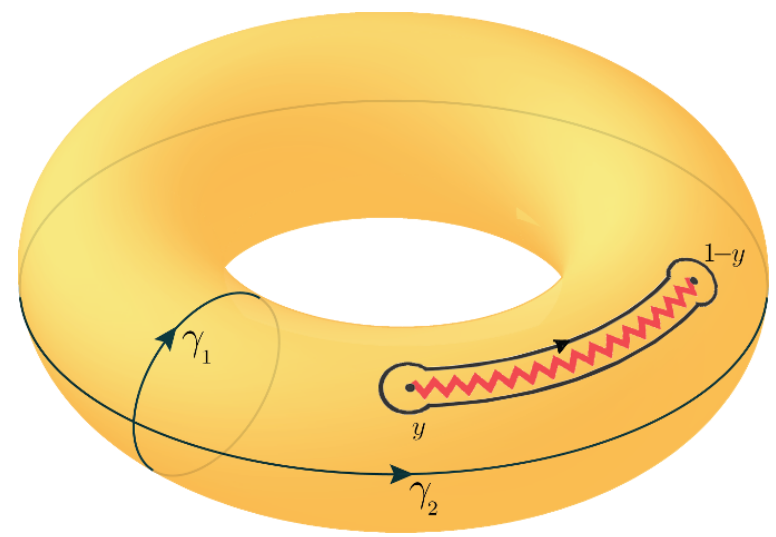

Figure 5. The deformable contour on the torus.

Note that $z$ is a real variable. The second integral vanishes as we had just shown. Using the double periodicity property $\vartheta_{1}(z)=\vartheta_{1}(z+1)$, the third integral can be set from $-y$ to 0 . So, we have

$$
W_{1}^{1}=\left(\int_{0}^{y}+\int_{-y}^{0}\right) d z \vartheta_{1}(z-y)^{-(1-k / n)} \vartheta_{1}(z-1+y)^{-k / n} \vartheta_{1}\left(z-y_{M}\right) .
$$

Similarly,

$$
W_{1}^{2}=\left(\int_{0}^{y}+\int_{-y}^{0}\right) d \bar{z} \vartheta_{1}(\bar{z}-y)^{-k / n} \vartheta_{1}(\bar{z}-1+y)^{-(1-k / n)} \vartheta_{1}\left(\bar{z}+y_{M}\right) .
$$

Again $\bar{z}$ is real. On changing variables in the above integral $\bar{z}=-z$ and using the fact that $\vartheta_{1}(-z)=-\vartheta_{1}(z)$ it can be seen that

$$
W_{1}^{1}=W_{1}^{2}
$$

We have thus proved the first two relations in (B.1).

Let us now consider the integrals over the temporal cycle. The integration is now over a purely imaginary variable. We also have $\tau=i \beta$.

$$
W_{2}^{1}=\int_{0}^{\tau} d z \vartheta_{1}(z-y)^{-(1-k / n)} \vartheta_{1}(z-1+y)^{-k / n} \vartheta_{1}\left(z-y_{M}\right) .
$$

Changing variables $z=i w(w$ is real), we have

$$
W_{2}^{1}=\int_{0}^{\beta} i d w \vartheta_{1}(i w-y)^{-(1-k / n)} \vartheta_{1}(i w-1+y)^{-k / n} \vartheta_{1}\left(i w-y_{M}\right) .
$$

On complex conjugation this becomes

$$
\left(W_{2}^{1}\right)^{*}=\int_{0}^{\beta}-i d w \vartheta_{1}(-i w-y)^{-(1-k / n)} \vartheta_{1}(-i w-1+y)^{-k / n} \vartheta_{1}\left(-i w-y_{M}\right) .
$$


On changing variables again $u=-w$ we get

$$
\left(W_{2}^{1}\right)^{*}=\int_{0}^{-\beta} i d u \vartheta_{1}(i u-y)^{-(1-k / n)} \vartheta_{1}(i u-1+y)^{-k / n} \vartheta_{1}\left(i u-y_{M}\right) .
$$

We now use the property, $\vartheta_{1}(i(u+\beta))=\vartheta_{1}(i u)$ to get

$$
\begin{aligned}
\left(W_{2}^{1}\right)^{*} & =\int_{\beta}^{0} i d u \vartheta_{1}(i u-y)^{-(1-k / n)} \vartheta_{1}(i u-1+y)^{-k / n} \vartheta_{1}\left(i u-y_{M}\right) \\
& =-W_{2}^{1} .
\end{aligned}
$$

On performing the same analysis it can be shown that $\left(W_{2}^{2}\right)^{*}=-W_{2}^{2}$.

Now let's consider the following integral

$$
W_{2}^{2}=\int_{0}^{\tau} d \bar{z} \vartheta_{1}(z-y)^{-k / n} \vartheta_{1}(\bar{z}-1+y)^{-(1-k / n)} \vartheta_{1}\left(\bar{z}+y_{M}\right) .
$$

Changing variables $z=i w$ or $\bar{z}=-i w$, with $w$ being real, we get

$$
W_{2}^{2}=\int_{0}^{\beta}-i d w \vartheta_{1}(-i w-y)^{-k / n} \vartheta_{1}(-i w-1+y)^{-(1-k / n)} \vartheta_{1}\left(-i w+y_{M}\right)
$$

Using $\vartheta_{1}(-z)=-\vartheta_{1}(z)$, we get

$$
\begin{aligned}
W_{2}^{2} & =-\int_{0}^{\beta} i d w \vartheta_{1}(i w+y)^{-k / n} \vartheta_{1}(i w+1-y)^{-(1-k / n)} \vartheta_{1}\left(i w-y_{M}\right) \\
& =-W_{2}^{1}=\left(W_{2}^{1}\right)^{*}
\end{aligned}
$$

The second and third equalities follow from (B.21). This completes the proofs of all relations in (B.1).

\section{Relating $W_{1}^{1}$ to $W_{2}^{2}$}

In this subsection we shall relate $W_{1}^{1}$ and $W_{2}^{2}$. Choosing the interval as $\left[\frac{1-L}{2}, \frac{1+L}{2}\right]$ the expressions are

$$
\begin{aligned}
& W_{1}^{1}=\int_{0}^{1} d z \frac{\vartheta_{1}(z-1 / 2+L / 2-(k L) / n)}{\vartheta_{1}(z-1 / 2+L / 2)^{1-k / n} \vartheta_{1}(z-1 / 2-L / 2)^{k / n}}, \\
& W_{2}^{2}=\int_{0}^{\tau} d \bar{z} \frac{\vartheta_{1}(\bar{z}-1 / 2-L / 2+(k L) / n)}{\vartheta_{1}(\bar{z}-1 / 2-L / 2)^{1-k / n} \vartheta_{1}(\bar{z}-1 / 2+L / 2)^{k / n}} .
\end{aligned}
$$

The integrands of the above are clearly related by $L \rightarrow-L$. Defining

$$
w(z, L)=\frac{\vartheta_{1}(z-1 / 2-L / 2-(k L) / n)}{\vartheta_{1}(z-1 / 2+L / 2)^{1-k / n} \vartheta_{1}(z-1 / 2-L / 2)^{k / n}},
$$

we can write

$$
W_{1}^{1}=\int_{0}^{1} d z w(z, L), \quad W_{2}^{2}=\int_{0}^{\tau} d \bar{z} w(\bar{z},-L) .
$$


Since $w(z, L)$ is constructed purely of theta functions, motivated by (A.1) let us now consider its Fourier series expansion

$$
w(z, L)=a_{0}(L)+\sum_{m \neq 0} a_{m}(L) e^{2 \pi i m z} .
$$

It is assumed that $a_{m}$ has an implicit $\tau$ dependence.

It can be seen from (B.27) that the function $w(z, L)$ is invariant under $z \rightarrow z+\tau$. We thus have the following identity relating the Fourier coefficients.

$$
\sum_{m \neq 0} a_{m}(L) e^{2 \pi i m z}=\sum_{m \neq 0} a_{m}(L) e^{2 \pi i m(z+\tau)} .
$$

Performing a indefinite integral over $z$ to the above relation, we get

$$
\sum_{m \neq 0} \frac{a_{m}(L) e^{2 \pi i m z}}{2 \pi i m}=\sum_{m \neq 0} \frac{a_{m}(L) e^{2 \pi i m(z+\tau)}}{2 \pi i m} .
$$

The form of the integral for $W_{2}^{2}$ is

$$
\begin{aligned}
W_{2}^{2}=\int_{0}^{\tau} d z w(z,-L) & =\int_{0}^{\tau} d z\left[a_{0}(-L)+\sum_{m \neq 0} a_{m}(-L) e^{2 \pi i m z}\right] \\
& =\left[a_{0}(-L) z+\sum_{m \neq 0} \frac{a_{m}(-L) e^{2 \pi i m z}}{2 \pi i m}\right]_{0}^{\tau},
\end{aligned}
$$

which upon using (B.31) is simply

$$
W_{2}^{2}=a_{0}(-L) \tau
$$

One can however perform an explicit integration of $W_{2}^{2}$ order by order in $q\left(=e^{-2 \pi / \beta}\right)$ and then verify that $W_{2}^{2}$ is an even function in $L$ (see equation (B.47) below). We therefore conclude that $a_{0}(L)=a_{0}(-L)$ and

$$
W_{2}^{2}=a_{0}(L) \tau
$$

Similarly, the $W_{1}^{1}$ integral (B.28) can be easily done and we get

$$
W_{1}^{1}=a_{0}(L)
$$

Comparing (B.34) and (B.35) we have the following relation

$$
W_{2}^{2}=\tau W_{1}^{1}
$$

The expression for $W_{2}^{2}$ can be also related to the $W_{1}^{1}$ in another way.

$$
W_{2}^{2}=\int_{0}^{\tau} d \bar{z} \frac{\vartheta_{1}(\bar{z}-1 / 2-L / 2+k L / n)}{\vartheta_{1}(\bar{z}-1 / 2+L / 2)^{k / n} \vartheta_{1}(\bar{z}-1 / 2-L / 2)^{(1-k / n)}} .
$$


Performing a S-modular transformation to the above integrand we get

$$
W_{2}^{2}=e^{\frac{\pi L^{2}}{\beta} \frac{k}{n}\left(1-\frac{k}{n}\right)} \int_{0}^{\tau} d \bar{z} \frac{\vartheta_{1}\left(\frac{\bar{z}-1 / 2-L / 2+k L / n}{\tau} \mid-\frac{1}{\tau}\right)}{\vartheta_{1}\left(\frac{\bar{z}-1 / 2+L / 2}{\tau} \mid-\frac{1}{\tau}\right)^{k / n} \vartheta_{1}\left(\frac{\bar{z}-1 / 2-L / 2}{\tau} \mid-\frac{1}{\tau}\right)^{(1-k / n)}} .
$$

Now, changing the integration variable from $\bar{z}$ to $w=-\bar{z} / \tau$ and then shifting the range of integration from $[-1,0]$ to $[0,1]$ (which is allowed by periodicity of the integrand) we get

$$
W_{2}^{2}=\tau e^{\frac{\pi L^{2}}{\beta} \frac{k}{n}\left(1-\frac{k}{n}\right)} \int_{0}^{1} d w \frac{\vartheta_{1}\left(w+\frac{1 / 2+L / 2-k L / n}{\tau} \mid-\frac{1}{\tau}\right)}{\vartheta_{1}\left(w+\frac{1 / 2-L / 2}{\tau} \mid-\frac{1}{\tau}\right)^{k / n} \vartheta_{1}\left(w+\frac{1 / 2+L / 2}{\tau} \mid-\frac{1}{\tau}\right)^{(1-k / n)}} .
$$

We can now use the periodicity properties of $\vartheta_{1}(z / \tau \mid-1 / \tau)$ and shift the argument by $-1 / \tau$ in the above integrand to get

$$
W_{2}^{2}=\tau e^{\frac{\pi L^{2}}{\beta} \frac{k}{n}\left(1-\frac{k}{n}\right)} \int_{0}^{1} d w \frac{\vartheta_{1}\left(w+\frac{-1 / 2+L / 2-k L / n}{\tau} \mid-\frac{1}{\tau}\right)}{\vartheta_{1}\left(w+\frac{-1 / 2-L / 2}{\tau} \mid-\frac{1}{\tau}\right)^{k / n} \vartheta_{1}\left(w+\frac{-1 / 2+L / 2}{\tau} \mid-\frac{1}{\tau}\right)^{(1-k / n)}} .
$$

The integral appearing above is the that of $W_{1}^{1}$ with the replacements: $L \rightarrow L / \tau$ and $\tau=-1 / \tau$. We finally have the following result ${ }^{10}$

$$
W_{2}^{2}(L, \tau)=\tau e^{\frac{\pi L^{2}}{\beta} \frac{k}{n}\left(1-\frac{k}{n}\right)} W_{1}^{1}(L / \tau,-1 / \tau) .
$$

\section{High temperature expansions of $W_{1}^{1}$ and $W_{2}^{2}$}

We have

$$
W_{2}^{2}=\int_{0}^{\tau} d \bar{z} \frac{\vartheta_{1}\left(\bar{z}-\frac{1}{2}-\frac{L}{2}+\frac{k L}{n}\right)}{\vartheta_{1}\left(\bar{z}-\frac{1}{2}+\frac{L}{2}\right)^{k / n} \vartheta_{1}\left(\bar{z}-\frac{1}{2}-\frac{L}{2}\right)^{1-k / n}}
$$

Using the periodicity property of the $\vartheta_{1}(z \mid \tau)$

$$
\vartheta_{2}(z \mid \tau)=-\vartheta_{1}(z-1 / 2 \mid \tau)
$$

we get

$$
W_{2}^{2}=\int_{0}^{\tau} d \bar{z} \frac{\vartheta_{2}\left(\bar{z}-\frac{L}{2}+\frac{k L}{n}\right)}{\vartheta_{2}\left(\bar{z}+\frac{L}{2}\right)^{k / n} \vartheta_{2}\left(\bar{z}-\frac{L}{2}\right)^{1-k / n}} .
$$

Performing a S-modular transformation to the above integrand

$$
W_{2}^{2}=e^{\frac{\pi}{\beta} \frac{k}{n}\left(1-\frac{k}{n}\right) L^{2}} \int_{0}^{\tau} d \bar{z} \frac{\vartheta_{4}\left(\frac{\bar{z}-L / 2+k L / n}{\tau} \mid-\frac{1}{\tau}\right)}{\vartheta_{4}\left(\frac{\bar{z}+L / 2}{\tau} \mid-\frac{1}{\tau}\right)^{k / n} \vartheta_{4}\left(\frac{\bar{z}-L / 2}{\tau} \mid-\frac{1}{\tau}\right)^{1-k / n}} .
$$

\footnotetext{
${ }^{10}$ In the arguments of $\vartheta_{1}$ in the integrand of $W_{1}^{1} 1 / 2$ gets replaced by $1 /(2 \tau)$. This can be thought of as the length of the spatial circle $R$ being replaced by $R / \tau$.
} 
Changing variables to $w$, where $z=i w$ and $w \in \mathbb{R}$ since the integral is along the negative imaginary axis

$$
W_{2}^{2}=-i e^{\frac{\pi}{\beta} \frac{k}{n}\left(1-\frac{k}{n}\right) L^{2}} \int_{0}^{-\beta} d w \frac{\vartheta_{4}\left(\frac{-i w-L / 2+k L / n}{\tau} \mid-\frac{1}{\tau}\right)}{\vartheta_{4}\left(\frac{-i w+L / 2}{\tau} \mid-\frac{1}{\tau}\right)^{k / n} \vartheta_{4}\left(\frac{-i w-L / 2}{\tau} \mid-\frac{1}{\tau}\right)^{1-k / n}} .
$$

We can now expand the above integral in powers of $q=e^{-2 \pi / \beta}$,

$$
\begin{aligned}
& \quad W_{2}^{2}(k, n) \\
& =e^{\frac{\pi}{\beta} \frac{k}{n}\left(1-\frac{k}{n}\right) L^{2} \tau} \\
& \times\left[1-\frac{2}{n^{2}}\left(-k^{2}+k n \cosh \left(\frac{2 \pi L(n-k)}{n \beta}\right)+(k-n)\left(k \cosh \left(\frac{2 \pi L}{\beta}\right)-n \cosh \left(\frac{2 \pi k L}{n \beta}\right)\right)+k n-n^{2}\right) q^{2}\right. \\
& +\frac{1}{2 n^{4}}\left(2 n \left(3(k-n)\left(k^{2}-k n+2 n^{2}\right) \cosh \left(\frac{2 \pi k L}{n}\right)\right.\right. \\
& +k\left(-3\left(k^{2}-k n+2 n^{2}\right) \cosh \left(\frac{2 \pi L(n-k)}{n}\right)-(k-2 n)(k-n) \cosh \left(\frac{2 \pi L(k+n)}{n}\right)\right. \\
& \left.\left.\quad+(k-n)(k+n) \cosh \left(\frac{2 \pi L(k-2 n)}{n}\right)\right)\right) \\
& \quad+4 k(k-n)\left(k^{2}-k n+4 n^{2}\right) \cosh \left(\frac{2 \pi L}{\beta}\right)+3\left(k^{4}-2 k^{3} n+7 k^{2} n^{2}-6 k n^{3}+4 n^{4}\right) \\
& \left.\left.+k(k-n)(k+n)(k-2 n) \cosh \left(\frac{4 \pi L}{\beta}\right)\right) q^{4}+\mathcal{O}\left(q^{6}\right)\right] .
\end{aligned}
$$

As remarked earlier it can be clearly seen that the above expression is a even function in $L$. The high-temperature expansion of $W_{1}^{1}$ can be obtained by using the relation (B.36).

\section{$W_{1}^{1}$ and $W_{2}^{2}$ in the $y \rightarrow 0$ limit}

Using the results of the previous subsections above we shall exactly evaluate the integrals $W_{1}^{1}$ and $W_{2}^{2}$ in the large interval or $y \rightarrow 0$ limit. For $y=\epsilon$ we have

$$
W_{1}^{1}=\int_{0}^{1} d z \vartheta_{1}(z-\epsilon)^{-(1-k / n)} \vartheta_{1}(z-1+\epsilon)^{-k / n} \vartheta_{1}\left(z-y_{M}\right)
$$

here, $y_{M}=\left(1-\frac{2 k}{n}\right) \epsilon+\frac{k}{n}$. The above integral can be written as

$$
W_{1}^{1}=\left(\int_{0}^{\epsilon}+\int_{\epsilon}^{1-\epsilon}+\int_{1-\epsilon}^{1}\right) d z \vartheta_{1}(z-\epsilon)^{-(1-k / n)} \vartheta_{1}(z-1+\epsilon)^{-k / n} \vartheta_{1}\left(z-y_{M}\right)
$$

The branch cut here is in the interval $[\epsilon, 1-\epsilon]$. It was seen in (B.11) that there is no contribution to the integral from this region. We thus have

$$
\begin{aligned}
W_{1}^{1} & =\left(\int_{0}^{\epsilon}+\int_{1-\epsilon}^{1}\right) d z \vartheta_{1}(z-\epsilon)^{-(1-k / n)} \vartheta_{1}(z-1+\epsilon)^{-k / n} \vartheta_{1}\left(z-y_{M}\right) \\
& =2 \int_{0}^{\epsilon} d z \frac{\vartheta_{1}\left(z-y_{M}\right)}{\vartheta_{1}(z-\epsilon)^{(1-k / n)} \vartheta_{1}(z-1+\epsilon)^{k / n}} \\
& \simeq \lim _{\epsilon \rightarrow 0} 2 \epsilon\left(\frac{1}{2} \frac{\vartheta_{1}(-k / n)}{\vartheta_{1}(\epsilon)}\right)=\frac{\vartheta_{1}(-k / n)}{\vartheta_{1}^{\prime}(0)} .
\end{aligned}
$$


Note that here we have used the fact that integrand is real in the interval $[0, \epsilon]$ and $[1-\epsilon, 1]$. We have multiplied the integral by $1 / 2$ to take into account the contribution per unit cell. Using (B.36) we have

$$
W_{2}^{2} \simeq \tau \frac{\vartheta_{1}(-k / n)}{\vartheta_{1}^{\prime}(0)}
$$

\section{One-loop determinant for the Chern-Simons gauge field}

In this subsection we shall calculate the one-loop determinant for the Chern-Simons spin-1 field. We present this calculation in detail since we did not find an explicit discussion of this in the literature. The one-loop determinant is given in terms of the product of the one-loop determinants of the scalar and the transverse vector Laplacian [44].

$$
Z_{\text {one-loop }}=\operatorname{det}^{1 / 2}\left(-\Delta_{(0)}\right) \operatorname{det}^{-1 / 4}\left(-\Delta_{A^{\perp}}-2\right) \text {. }
$$

The scalar and $A^{\perp}$ one-loop determinants are as follows

$$
\begin{aligned}
\frac{1}{2} \log \operatorname{det}\left(-\Delta_{(0)}\right) & =-\sum_{m=1}^{\infty} \frac{|q|^{2 m}}{m\left|1-q^{m}\right|^{2}}, \\
-\frac{1}{4} \log \operatorname{det}\left(-\Delta_{A^{\perp}}-2\right) & =\frac{1}{2} \sum_{m=1}^{\infty} \frac{q^{m}+\bar{q}^{m}}{m\left|1-q^{m}\right|} .
\end{aligned}
$$

Adding the contributions from the two terms above we get

$$
\begin{aligned}
\log Z_{\text {one-loop }} & =\sum_{m=1}^{\infty} \frac{1}{2 m\left|1-q^{m}\right|^{2}}\left(-2(q \bar{q})^{m}+q^{m}+\bar{q}^{m}\right) \\
& =\sum_{m=1}^{\infty} \frac{1}{2 m\left|1-q^{m}\right|^{2}}\left(2\left|1-q^{m}\right|^{2}-\left(1-q^{m}\right)-\left(1-\bar{q}^{m}\right)\right) \\
& =-\sum_{m=1}^{\infty} \frac{1}{m}+\frac{1}{2} \sum_{m=1}^{\infty} \frac{1}{m\left(1-q^{m}\right)}+\frac{1}{2} \sum_{m=1}^{\infty} \frac{1}{m\left(1-\bar{q}^{m}\right)} \\
& =\frac{1}{2} \sum_{n=1}^{\infty} \sum_{n=1}^{\infty} \frac{q^{n m}}{m}+\frac{1}{2} \sum_{n=1}^{\infty} \sum_{n=1}^{\infty} \frac{\bar{q}^{n m}}{m} \\
& =-\frac{1}{2} \sum_{n=1}^{\infty} \log \left|1-q^{n}\right|^{2} .
\end{aligned}
$$

So, the one-loop determinant of a single U(1) Chern-Simons gauge field is

$$
Z_{\text {one-loop }}=\prod_{n=1}^{\infty}\left(\frac{1}{\left|1-q^{n}\right|^{2}}\right)^{1 / 2} \text {. }
$$




\section{Sums involving 2-letter words}

We need to do the sums over $k_{1}$ and $k_{2}$ over the reciprocals of the eigenvalues of the 2-letter words given below.

$$
\begin{aligned}
& q_{ \pm \pm}^{-1 / 2}=\frac{n^{2}}{\left(u_{y}^{-1}-u_{y}\right)^{2} u_{R}}\left(u_{y}^{-1 / n}-e^{2 \pi i\left(k_{1}-k_{2}\right) / n} u_{y}^{1 / n}\right)\left(u_{y}^{-1 / n}-e^{-2 \pi i\left(k_{1}-k_{2}\right) / n} u_{y}^{1 / n}\right)+\mathcal{O}(1) \\
& q_{ \pm \mp}^{-1 / 2}=-\frac{n^{2}}{\left(u_{y}^{-1}-u_{y}\right)^{2} u_{R}}\left(1-e^{2 \pi i\left(k_{1}-k_{2}\right) / n}\right)\left(1-e^{-2 \pi i\left(k_{1}-k_{2}\right) / n}\right)+\mathcal{O}(1) .
\end{aligned}
$$

The first sum is of the following form

$$
\sum_{k_{1}=0}^{n-1} \sum_{k_{2}=0}^{n-1} q_{ \pm \pm}^{1 / 2}=\frac{4 \sinh ^{2}(2 \pi T y) e^{-2 \pi T R}}{n^{2} e^{4 \pi T y / n}} \sum_{k_{1}=0}^{n-1} \sum_{k_{2}=0}^{n-1} \frac{1}{\left(1-r e^{i\left(k_{1}-k_{2}\right) 2 \pi / n}\right)\left(1-r e^{-i\left(k_{1}-k_{2}\right) 2 \pi / n}\right)}
$$

where $r=u_{y}^{2 / n}$. Let us now do the sum above. Implementing the $k_{1} \neq k_{2}$ constraint by removing the 'diagonal' $k_{1}=k_{2}$ piece and counting $\left(k_{1}, k_{2}\right)$ pairs only once we get

$$
\begin{aligned}
& \frac{1}{2}\left(\sum_{k_{1}=0}^{n-1} \sum_{k_{2}=0}^{n-1} \frac{1}{\left(1-r e^{i\left(k_{1}-k_{2}\right) 2 \pi / n}\right)\left(1-r e^{-i\left(k_{1}-k_{2}\right) 2 \pi / n}\right)}-\frac{n}{(1-r)^{2}}\right) \\
= & \frac{1}{2}\left(\sum_{k_{1}=0}^{n-1} \sum_{k_{2}=0}^{n-1} \sum_{l=0}^{\infty} r^{l} e^{2 \pi i l\left(k_{1}-k_{2}\right) / n} \sum_{s=0}^{\infty} r^{s} e^{-2 \pi i s\left(k_{1}-k_{2}\right) / n}-\frac{n}{(1-r)^{2}}\right) \\
= & \frac{1}{2}\left(\sum_{l=0}^{\infty} \sum_{s=0}^{\infty} r^{l+s} \sum_{k_{1}=0}^{n-1} e^{2 \pi i k_{1}(l-s) / n} \sum_{k_{2}=0}^{n-1} e^{-2 \pi i k_{2}(l-s) / n}-\frac{n}{(1-r)^{2}}\right) .
\end{aligned}
$$

The sums over $k_{1,2}$ are non-zero only if $l-s=M n$ where $M \in \mathcal{Z}$. So, one can write the above sum as

$$
\frac{1}{2}\left(\sum_{l=0}^{\infty} \sum_{s=0}^{\infty} r^{l+s} \sum_{M=0}^{\infty}\left(n \delta_{l-s, M}\right)-\frac{n}{(1-r)^{2}}\right)
$$

where

$$
\begin{array}{ll}
l=M n+s, & s=N n+j \\
s \in\{0,1,2, \cdots\} & j \in\{0,1,2, \cdots, n-1\} \quad n \in\{0,1,2, \cdots\}
\end{array}
$$


Also, $l>0$ implies $M n+N n+j>0$, which means $M$ can take the lowest value $-N$. Converting the above sum over $l, s$ and $M$ to $N, M$ and $j$, we get

$$
\begin{aligned}
& \frac{1}{2}\left(n^{2} \sum_{N=0}^{\infty} \sum_{j=0}^{n-1} \sum_{M=-N}^{\infty} r^{M n+2(N n+j)}-n \sum_{l=0}^{\infty} \sum_{s=0}^{\infty} r^{l+s}\right) \\
= & \frac{1}{2}\left(n^{2} \sum_{N=0}^{\infty} \sum_{j=0}^{n-1} r^{2 j+2 N n} \sum_{M=-N}^{\infty} r^{M n}-\frac{n}{(1-r)^{2}}\right) \\
= & \frac{1}{2}\left(n^{2} \sum_{N=0}^{\infty} \sum_{j=0}^{n-1} r^{2 j+2 N n} \frac{r^{-N n}}{1-r^{n}}-\frac{n}{(1-r)^{2}}\right) \\
= & \frac{1}{2}\left(n^{2} \frac{1}{\left(1-r^{n}\right)^{2}} \frac{1-r^{2 n}}{1-r^{2}}-\frac{n}{(1-r)^{2}}\right) \\
= & \frac{1}{2}\left(n^{2} \frac{r^{-1}}{r^{-1}-r} \frac{r^{-n / 2}+r^{n / 2}}{r^{-n / 2}-r^{n / 2}}-\frac{n}{(1-r)^{2}}\right) \\
= & \frac{1}{2}\left(n^{2} \frac{e^{4 \pi T y / n}}{2 \sinh \left(\frac{4 \pi T y}{n}\right)} \operatorname{coth}(2 \pi T y)-n \frac{e^{4 \pi T y / n}}{4 \sinh ^{2}(2 \pi T y / n)}\right) .
\end{aligned}
$$

Using this in (D.3) we get

$$
\sum_{k_{1}=0}^{\infty} \sum_{k_{2}=0}^{\infty} q_{ \pm \pm}^{1 / 2}=\frac{\sinh \left(\frac{4 \pi T y}{n}\right)}{2 \sinh (4 \pi T y)} e^{-2 \pi T R}-\frac{\sinh ^{2}\left(\frac{2 \pi T y}{n}\right)}{2 n \sinh ^{2}(2 \pi T y)} e^{-2 \pi T R}+\mathcal{O}\left(e^{-4 \pi T R}\right) .
$$

The second sum (D.2) is

$$
\begin{aligned}
\sum_{k_{1}=0}^{\infty} \sum_{k_{2}=0}^{\infty} q_{ \pm \mp}^{1 / 2}=- & \frac{4 \sinh ^{2}(2 \pi T y) e^{-2 \pi T R}}{n^{2}} \\
& \times \lim _{r \rightarrow 1^{-}} \sum_{k_{1}=0}^{n-1} \sum_{k_{2}=0}^{\infty} \frac{1}{\left(1-r e^{i\left(k_{1}-k_{2}\right) 2 \pi / n}\right)\left(1-r e^{-i\left(k_{1}-k_{2}\right) 2 \pi / n}\right)}
\end{aligned}
$$

Let us now do the sum above. Taking into account the constraints which were present while doing the previous sum we have

$$
\begin{aligned}
& \frac{1}{2}\left(\sum_{k_{1}=0}^{n-1} \sum_{k_{2}=0}^{n-1} \frac{1}{\left(1-r e^{i\left(k_{1}-k_{2}\right) 2 \pi / n}\right)\left(1-r e^{-i\left(k_{1}-k_{2}\right) 2 \pi / n}\right)}-\frac{n}{(1-r)^{2}}\right) \\
= & \frac{1}{2}\left(\sum_{k_{1}=0}^{n-1} \sum_{k_{2}=0}^{n-1} \sum_{l=0}^{\infty} r^{l} e^{2 \pi i l\left(k_{1}-k_{2}\right) / n} \sum_{s=0}^{\infty} r^{s} e^{-2 \pi i s\left(k_{1}-k_{2}\right) / n}-\frac{n}{(1-r)^{2}}\right) \\
= & \frac{1}{2}\left(\sum_{l=0}^{\infty} \sum_{s=0}^{\infty} r^{l+s} \sum_{k_{1}=0}^{n-1} e^{2 \pi i k_{1}(l-s) / n} \sum_{k_{2}=0}^{n-1} e^{-2 \pi i k_{2}(l-s) / n}-\frac{n}{(1-r)^{2}}\right) \\
= & \frac{1}{2}\left(\sum_{l=0}^{\infty} \sum_{s=0}^{\infty} r^{l+s} \sum_{k_{1}=0}^{n-1} e^{2 \pi i k_{1}(l-s) / n} \sum_{k_{2}=0}^{n-1} e^{-2 \pi i k_{2}(l-s) / n}-\frac{n}{(1-r)^{2}}\right) .
\end{aligned}
$$


The first term in (D.10) is the same as the one calculated in the previous sum (D.7). So, the above sum becomes

$$
\frac{1}{2}\left(n^{2} \frac{1}{\left(1-r^{n}\right)^{2}} \frac{1-r^{2 n}}{1-r^{2}}-n \frac{1}{(1-r)^{2}}\right) .
$$

One can now take the limit $r \rightarrow 1^{-}$. We get

$$
\lim _{r \rightarrow 1^{-}} \frac{1}{2 n^{2}}\left[n^{2} \frac{1}{\left(1-r^{n}\right)^{2}} \frac{1-r^{2 n}}{1-r^{2}}-n \frac{1}{(1-r)^{2}}\right]=\frac{1}{24 n}\left(n^{2}-1\right) .
$$

Using the above in (D.9) we obtain

$$
\sum_{k_{1}=0}^{\infty} \sum_{k_{2}=0}^{\infty} q_{ \pm \mp}^{1 / 2}=-\frac{\left(n^{2}-1\right)}{6 n} \sinh ^{2}(2 \pi T y) e^{-2 \pi T R}+\mathcal{O}\left(e^{-4 \pi T R}\right)
$$

The above sums (D.8) and (D.13) are used in (5.35) in order to obtain (5.37).

Open Access. This article is distributed under the terms of the Creative Commons Attribution License (CC-BY 4.0), which permits any use, distribution and reproduction in any medium, provided the original author(s) and source are credited.

\section{References}

[1] A. Osterloh, L. Amico, G. Falci and R. Fazio, Scaling of entanglement close to a quantum phase transition, Nature 416 (2002) 608 [quant-ph/0202029].

[2] L. Amico, R. Fazio, A. Osterloh and V. Vedral, Entanglement in many-body systems, Rev. Mod. Phys. 80 (2008) 517 [quant-ph/0703044] [INSPIRE].

[3] P. Calabrese and J.L. Cardy, Evolution of entanglement entropy in one-dimensional systems, J. Stat. Mech. 0504 (2005) P04010 [cond-mat/0503393] [INSPIRE].

[4] L. Bombelli, R.K. Koul, J. Lee and R.D. Sorkin, A Quantum Source of Entropy for Black Holes, Phys. Rev. D 34 (1986) 373 [inSPIRE].

[5] M. Srednicki, Entropy and area, Phys. Rev. Lett. 71 (1993) 666 [hep-th/9303048] [INSPIRE].

[6] C. Holzhey, F. Larsen and F. Wilczek, Geometric and renormalized entropy in conformal field theory, Nucl. Phys. B 424 (1994) 443 [hep-th/9403108] [INSPIRE].

[7] P. Calabrese and J.L. Cardy, Entanglement entropy and quantum field theory, J. Stat. Mech. 0406 (2004) P06002 [hep-th/0405152] [INSPIRE].

[8] P. Calabrese and J. Cardy, Entanglement entropy and conformal field theory, J. Phys. A 42 (2009) 504005 [arXiv:0905.4013] [INSPIRE].

[9] H. Casini and M. Huerta, Entanglement entropy in free quantum field theory, J. Phys. A 42 (2009) 504007 [arXiv:0905.2562] [InSPIRE].

[10] P. Calabrese, J. Cardy and E. Tonni, Entanglement entropy of two disjoint intervals in conformal field theory, J. Stat. Mech. 0911 (2009) P11001 [arXiv: 0905. 2069] [inSPIRE].

[11] P. Calabrese, J. Cardy and E. Tonni, Entanglement entropy of two disjoint intervals in conformal field theory II, J. Stat. Mech. 1101 (2011) P01021 [arXiv:1011.5482] [INSPIRE]. 
[12] M. Headrick, Entanglement Rényi entropies in holographic theories, Phys. Rev. D 82 (2010) 126010 [arXiv: 1006.0047 ] [INSPIRE].

[13] A. Coser, L. Tagliacozzo and E. Tonni, On Rényi entropies of disjoint intervals in conformal field theory, J. Stat. Mech. 2014 (2014) P01008 [arXiv: 1309.2189] [INSPIRE].

[14] T. Azeyanagi, T. Nishioka and T. Takayanagi, Near Extremal Black Hole Entropy as Entanglement Entropy via $A d S_{2} / C F T_{1}$, Phys. Rev. D 77 (2008) 064005 [arXiv:0710. 2956] [INSPIRE].

[15] C.P. Herzog and T. Nishioka, Entanglement Entropy of a Massive Fermion on a Torus, JHEP 03 (2013) 077 [arXiv: 1301.0336] [INSPIRE].

[16] C.P. Herzog and M. Spillane, Tracing Through Scalar Entanglement, Phys. Rev. D 87 (2013) 025012 [arXiv: 1209.6368] [inSPIRE].

[17] L.J. Dixon, D. Friedan, E.J. Martinec and S.H. Shenker, The Conformal Field Theory of Orbifolds, Nucl. Phys. B 282 (1987) 13 [INSPIRE].

[18] J.J. Atick, L.J. Dixon, P.A. Griffin and D. Nemeschansky, Multiloop Twist Field Correlation Functions for $Z(N)$ Orbifolds, Nucl. Phys. B 298 (1988) 1 [inSPIRE].

[19] H. Saleur, Correlation functions of the critical Ashkin-Teller model on a torus, J. Stat. Phys. 50 (1988) 475.

[20] R. Dijkgraaf, E.P. Verlinde and H.L. Verlinde, $C=1$ Conformal Field Theories on Riemann Surfaces, Commun. Math. Phys. 115 (1988) 649 [InSPIRE].

[21] S. Ryu and T. Takayanagi, Holographic derivation of entanglement entropy from AdS/CFT, Phys. Rev. Lett. 96 (2006) 181602 [hep-th/0603001] [inSPIRE].

[22] S. Ryu and T. Takayanagi, Aspects of Holographic Entanglement Entropy, JHEP 08 (2006) 045 [hep-th/0605073] [INSPIRE].

[23] T. Faulkner, The Entanglement Rényi Entropies of Disjoint Intervals in AdS/CFT, arXiv:1303.7221 [INSPIRE].

[24] A. Lewkowycz and J. Maldacena, Generalized gravitational entropy, JHEP 08 (2013) 090 [arXiv: 1304.4926] [INSPIRE].

[25] K. Krasnov, Holography and Riemann surfaces, Adv. Theor. Math. Phys. 4 (2000) 929 [hep-th/0005106] [INSPIRE].

[26] T. Barrella, X. Dong, S.A. Hartnoll and V.L. Martin, Holographic entanglement beyond classical gravity, JHEP 09 (2013) 109 [arXiv:1306.4682] [INSPIRE].

[27] T. Faulkner, A. Lewkowycz and J. Maldacena, Quantum corrections to holographic entanglement entropy, JHEP 11 (2013) 074 [arXiv:1307.2892] [INSPIRE].

[28] B. Swingle, L. Huijse and S. Sachdev, Entanglement entropy of compressible holographic matter: loop corrections from bulk fermions, arXiv:1308.3234 [INSPIRE].

[29] B. Chen and J.-J. Zhang, On short interval expansion of Rényi entropy, JHEP 11 (2013) 164 [arXiv: 1309.5453] [INSPIRE].

[30] X. Yin, Partition Functions of Three-Dimensional Pure Gravity, Commun. Num. Theor. Phys. 2 (2008) 285 [arXiv:0710.2129] [INSPIRE].

[31] S. Giombi, A. Maloney and X. Yin, One-loop Partition Functions of 3D Gravity, JHEP 08 (2008) 007 [arXiv:0804.1773] [INSPIRE]. 
[32] N.J. Sloane et al., Sphere packings, lattices and groups, vol. 290, pg. 108-110, Springer (1999).

[33] P.H. Ginsparg, Applied conformal field theory, hep-th/9108028 [INSPIRE].

[34] E. Perlmutter, A universal feature of CFT Rényi entropy, JHEP 03 (2014) 117 [arXiv: 1308.1083] [INSPIRE].

[35] A. Maloney and E. Witten, Quantum Gravity Partition Functions in Three Dimensions, JHEP 02 (2010) 029 [arXiv:0712.0155] [INSPIRE].

[36] M.R. Gaberdiel, R. Gopakumar and A. Saha, Quantum W-symmetry in AdS $S_{3}$, JHEP 02 (2011) 004 [arXiv: 1009.6087] [INSPIRE].

[37] J.R. David, M.R. Gaberdiel and R. Gopakumar, The Heat Kernel on AdS $S_{3}$ and its Applications, JHEP 04 (2010) 125 [arXiv:0911.5085] [INSPIRE].

[38] J.R. David, M. Gaberdiel and R. Gopakumar, Heat kernels on $A d S_{3}$, unpublished notes.

[39] S. Datta and J.R. David, Higher spin fermions in the BTZ black hole, JHEP 07 (2012) 079 [arXiv: 1202.5831] [INSPIRE].

[40] M.A. Cazalilla, R. Citro, T. Giamarchi, E. Orignac and M. Rigol, One dimensional bosons: From condensed matter systems to ultracold gases, Rev. Mod. Phys. 83 (2011) 1405 [arXiv:1101.5337].

[41] T. Hartman, Entanglement Entropy at Large Central Charge, arXiv:1303.6955 [INSPIRE].

[42] P. Caputa, G. Mandal and R. Sinha, Dynamical entanglement entropy with angular momentum and $\mathrm{U}(1)$ charge, JHEP 11 (2013) 052 [arXiv: 1306.4974] [INSPIRE].

[43] A. Belin et al., Holographic Charged Rényi Entropies, JHEP 12 (2013) 059 [arXiv: 1310.4180] [INSPIRE].

[44] J. Gegenberg and G. Kunstatter, The partition function for topological field theories, Annals Phys. 231 (1994) 270 [hep-th/9304016] [INSPIRE]. 